TRANSACTIONS OF THE

AMERICAN MATHEMATICAL SOCIETY

Volume 351, Number 2, February 1999, Pages 429-475

S 0002-9947(99)02335-1

\title{
QUADRATIC HOMOLOGY
}

\author{
HANS-JOACHIM BAUES
}

\begin{abstract}
We describe axioms for a 'quadratic homology theory' which generalize the classical axioms of homology. As examples we consider quadratic homology theories induced by 2-excisive homotopy functors in the sense of Goodwillie and the homology of a space with coefficients in a square group which generalizes the homology of a space with coefficients in an abelian group.
\end{abstract}

\section{INTRODUCTION}

The development of algebraic topology was profoundly affected by the notion of homology. Originally homology had coefficients in abelian groups and EilenbergSteenrod described the axioms of such an ordinary homology theory. Somewhat later important examples of generalized homology theories were found which led to the notion of homology with coefficients in a spectrum. The spectrum-homology can also be described as a homology theory satisfying all Eilenberg-Steenrod axioms except the dimension axiom concerning the value of the homology on spheres. In fact this value characterizes a homology theory in the sense that a natural transformation of homology theories which is an isomorphism on spheres is also an isomorphism on all finite CW-complexes.

In his recent work on the calculus of homotopy functors Goodwillie observed that a spectrum is equivalent to a linear homotopy functor and spectrum-homology of a space $X$ can be equivalently described by the homotopy groups.

$$
H_{n}(X, L)=\pi_{n} L(X)
$$

where $L$ is a linear homotopy functor. We therefore call spectrum-homology also a linear homology theory. The non-linear homology theories are then obtained by the homotopy groups

$$
H_{n}(X, D)=\pi_{n} D(X)
$$

where $D$ is any homotopy functor. Hence we again generalize homology by choosing now homotopy functors as coefficients. Clearly this is a very far reaching generalization since in particular homotopy groups of a space are the homology groups with coefficients in the identity functor. It is an old problem to find axioms which characterize the theory of homotopy groups in a similar way as ordinary homology theory is characterized by the Eilenberg-Steenrod axioms. The identity functor is

Received by the editors October 22, 1996.

1991 Mathematics Subject Classification. Primary 55N35, 55Q70, 55S20.

Key words and phrases. Quadratic functors, Goodwillie calculus, Steenrod squares, EHPsequence.

(C)1999 American Mathematical Society 
not linear but is still an analytic functor in the sense that there is a Taylor tower, $n \geq 1$,

$$
X \rightarrow P_{n}(X) \rightarrow P_{n-1}(X) \rightarrow \ldots \rightarrow P_{2}(X) \rightarrow P_{1}(X)
$$

approximating $X$. Here $P_{1}$ is linear, $P_{2}$ is quadratic and more generally $P_{n}$ is a reduced and $n$-excisive homotopy functor; compare [19,20]. A reduced $n$-excisive homotopy functor from spaces to spaces is the topological analogue of a polynomial functor of degree $n$ in algebra. The linear functor $P_{1}$ yields the homology theory of stable homotopy groups $\pi_{n}^{S}(X)=H_{n}\left(X, P_{1}\right)$.

In this paper we study as a first step outside the linear world the quadratic homology theories $H_{n}(X, Q)$ obtained by a quadratic homotopy functor $Q$. We introduce axioms of a quadratic homology theory such that $H_{n}(X, Q)$ satisfies these axioms. As in the classical case our axioms characterize a quadratic homology theory in the sense that a natural transformation between theories which is an isomorphism on spheres is also an isomorphism on all finite $\mathrm{CW}$-complexes. We deduce from the axioms various facts like the general EHP-sequence in $\S 3$. For example quadratic homotopy groups

$$
\pi_{n}^{Q}(X)=H_{n}\left(X, P_{2}\right)
$$

defined by $P_{2}$ in the Taylor tower (3) satisfy all the axioms. This is the quadratic analogue of stable homotopy groups.

The main purpose of this paper is a first discussion of basic axioms of a "quadratic homology theory" and the computation of examples obtained by "square homology". A problem like the quadratic analogue of Brown's representability theorem remains open; see the remark following (4.7).

Let $\mathbf{G r}$ be the category of groups. Then any group functor $F: \mathbf{G r} \rightarrow \mathbf{G r}$ as considered in (5.3) below induces a homotopy functor $F_{\sharp}$ which carries connected spaces to connected spaces. If $F$ is linear, that is, if the cross effect of $F$ is trivial (see 2.9) then $F(G)=G^{a b} \otimes A$ is given by an abelian group $A$ and ordinary homology with coefficients in $A$ can be described by

$$
\tilde{H}_{n}(X, A)=H_{n}\left(X, F_{\sharp}\right) \text {. }
$$

If the group functor $F$ is quadratic, that is, if the cross effect of $F$ is bilinear (see (2.9)) then we know by [10] that $F(G)=G \otimes M$ is given by a square group $M$ which is the quadratic analogue of an abelian group. We prove that then $F_{\sharp}$ is a quadratic homotopy functor. Hence the square homology

$$
H_{n}(X, M)=H_{n}\left(X, F_{\sharp}\right)
$$

with coefficients in a square group $M$ is a quadratic homology theory which generalizes ordinary homology. Using the Taylor tower of $F_{\sharp}$ we obtain the linearization

$$
F_{\sharp}=P_{2} F_{\sharp} \rightarrow P_{1} F_{\sharp}=F_{\sharp}^{l i n}
$$

which gives us a linear homology theory

$$
H_{n}^{S}(X, M)=H_{n}\left(X, F_{\sharp}^{l i n}\right)
$$

termed stable square-homology. Here $F_{\sharp}^{\text {lin }}$ corresponds to a spectrum $E_{M}$ so that we get a functor from the category of square groups to the homotopy category of spectra which carries $M$ to $E_{M}$. We show that $E_{M}$ is always the cofiber of a map $S q_{M}$ which carries an Eilenberg-Mac Lane spectrum to a product of Eilenberg-Mac 
Lane spectra. We call $S q_{M}$ the squaring operation associated to $M$. For example all Steenrod squares $S q^{2}, S q^{3}, \ldots$ can be derived from $S q_{M}$.

Let $\Gamma_{n} G$ and $\bar{\Gamma}_{n} G$ be the subgroups of a group $G$ given by the lower central series and the mod-2 restricted lower central series respectively. Then we obtain the quadratic group functors

$$
n i l_{2}, \overline{n i l}_{2}: \mathbf{G r} \rightarrow \mathbf{G r}
$$

which carry $G$ to the quotients $G / \Gamma_{3} G$ and $G / \bar{\Gamma}_{3} G$ respectively. The corresponding square groups are $\mathbb{Z}_{\text {nil }}$ and $\mathbb{Z}_{\text {nil }}^{4,2}$ with

$$
\begin{aligned}
& \operatorname{nil}_{2}(G)=G \otimes \mathbb{Z}_{n i l}, \\
& \overline{n i l}_{2}(G)=G \otimes \mathbb{Z}_{\text {nil }}^{4,2} .
\end{aligned}
$$

We compute the squaring operation $S q_{M}$ for $M=\mathbb{Z}_{n i l}$ and $M=\mathbb{Z}_{\text {nil }}^{4,2}$ explicitly in terms of Steenrod squares. This determines the spectrum associated to the linearization of the homotopy functor $\left(n i l_{2}\right)_{\sharp}$, resp. $\left(\overline{n i l}_{2}\right)_{\sharp}$; see $(8.16),(8.17)$.

The author happily acknowledges helpful conversations with Teimuraz Pirashvili. He also thanks G. Arone and T. Goodwillie for comments concerning "Calculus".

\section{CW-SpaCES, CW-PAirs AND SPECTRA}

We use the following conventions: Top is the category of topological spaces and Top* is the category of topological spaces with base point. We obtain cofibrations in Top by the universal homotopy extension property defined via the cylinder $I \times X$ where $I=[0,1]$ is the unit interval. A homotopy in Top* is a pointed map $I(X) \rightarrow Y$ where $I(X)=I \times X / I \times\{*\}$ is the reduced cylinder. By the inclusion $\left(i_{0}, i_{1}\right): X \vee X \subset I(X)$ we obtain the cone $C X=I(X) / i_{1} X$ and the suspension $\Sigma X=C X / i_{0} X$. Here we use the quotient space $X / Y$ which is defined for any pair of spaces $(X, Y)$ by the adjunction $X / Y=X \cup_{Y}\{*\}$. More generally an adjunction space $X \cup_{Y} Z$ is defined by a push out diagram

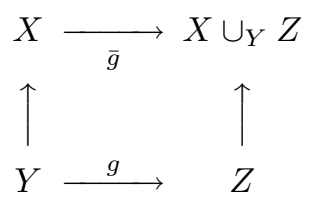

If $(X, Y)$ is a pair of spaces we call $\bar{g}:(X, Y) \rightarrow\left(X \cup_{Y} Z, Z\right)$ an adjunction map.

A space $X$ is a $C W$-space if there is a CW-complex $Y$ together with a homotopy equivalence $Y \simeq X$ in Top. The space $X$ is a finite CW-space if $Y$ can be chosen to be a CW-complex with finitely many cells. Moreover we write $\operatorname{dim}(X) \leq n$ if $\operatorname{dim}(Y) \leq n$. A $C W$-pair $(X, Y)$ is a pair in Top of CW-spaces $X$ and $Y$ for which the inclusion $Y \subset X$ is a cofibration. The pair is a finite CW-pair if $X$ and $Y$ are finite $\mathrm{CW}$-spaces. A CW-space $X$ is well pointed if the inclusion $\{*\} \rightarrow X$ is a cofibration.

Let space $\subset$ Top* be the full subcategory of well pointed CW-spaces and pointed maps. Moreover let pair be the category of well pointed $\mathrm{CW}$-pairs and pointed pair maps. We have functors

$$
\text { space } \rightarrow \text { pair } \rightarrow \text { space }
$$

which carry the space $X$ to the pair $(X, *)$ and carry the pair $(X, Y)$ to the quotient space $X / Y$. We also use the full subcategory space $_{r}$ of $(r-1)$-connected objects 
in space and the full subcategory pair ${ }_{r}$ of objects $(X, Y)$ in pair for which $X$ and $Y$ are $(r-1)$-connected. We point out that the suspension yields a functor

$$
\Sigma: \text { space }_{r} \rightarrow \text { space }_{r+1}
$$

raising the degree of connectedness.

A spectrum $E$ is a sequence of maps $\varepsilon_{n}: \Sigma E_{n} \rightarrow E_{n+1}$ in space, $n \in \mathbb{Z}$. A map $f: E \rightarrow E^{\prime}$ between spectra is a sequence of maps $f_{n}: E_{n} \rightarrow E_{n}^{\prime}$ with $f_{n+1} \varepsilon_{n}=\varepsilon_{n}^{\prime}\left(\Sigma f_{n}\right)$. Let spectra be the category of such spectra and maps.

A map $f: X \rightarrow Y$ in Top is a weak equivalence if $f$ induces a bijection of homotopy groups $f_{*}: \pi_{n} X \approx \pi_{n} Y, n \geq 0$, for every basepoint in the domain. A map in Top* is a weak equivalence if it is one in Top. Clearly a weak equivalence in space or pair is also a homotopy equivalence. A map $f: E \rightarrow E^{\prime}$ in spectra is a weak equivalence if it induces an isomorphism $f_{*}: \pi_{k} E \approx \pi_{k} E^{\prime}$. Here $\pi_{k} E=$ $\operatorname{colim}\left\{\pi_{n+k} E_{n}\right\}$ is an abelian group for all $k \in \mathbb{Z}$.

\section{Axioms of homology}

To fix notation we recall the following basic properties of a homology theory.

(2.1) Definition. Let $r \geq 0$. A suspension theory $(H, s)$ is a sequence of covariant functors, $n \in \mathbb{Z}$,

$$
H_{n}: \text { space }_{r} \rightarrow \mathbf{A b}
$$

together with a sequence of natural transformations, $n \in \mathbb{Z}$,

$$
s_{n}: H_{n} \rightarrow H_{n+1} \circ \Sigma
$$

such that $H_{n}(*)=0$ and $H_{n}\left(f_{0}\right)=H_{n}\left(f_{1}\right)$ for homotopic maps $f_{0} \simeq f_{1}$. We also consider suspension theories which satisfy some of the following axioms.

Exactness. For $(X, Y) \in$ pair $_{r}$ the sequence

$$
H_{n}(Y) \stackrel{i_{*}}{\longrightarrow} H_{n}(X) \stackrel{q_{*}}{\longrightarrow} H_{n}(X / Y)
$$

is exact where $i: Y \rightarrow X$ is the inclusion and $q: X \rightarrow X / Y$ is the quotient map.

Suspension. For $X \in$ space $_{r}$ the natural map

$$
s_{n}(X): H_{n}(X) \rightarrow H_{n+1}(\Sigma X)
$$

given by the transformation $s_{n}$ is an isomorphism for all $n \in \mathbb{Z}$.

Colimit axiom. For each sequence $X_{0} \longmapsto X_{1} \longmapsto \ldots$ of cofibrations in space ${ }_{r}$ the induced map

$$
\operatorname{colim}\left\{H_{n}\left(X_{i}\right)\right\} \rightarrow H_{n}\left(\operatorname{colim}\left\{X_{i}\right\}\right)
$$

is an isomorphism.

A suspension theory $(H, s)$ is termed a homology theory on space ${ }_{r}$ if the exactness and suspension axioms are satisfied; compare [29].

Given a supension theory $(H, s)$ we obtain the stable theory $\left(H^{S}, s\right)$ associated to $(H, s)$. Here $H_{n}^{S}(X)$ is the colimit of

$$
H_{n}(X) \stackrel{s}{\longrightarrow} H_{n+1}(\Sigma X) \rightarrow \ldots \rightarrow H_{n+k}\left(\Sigma^{k} X\right) \stackrel{s}{\longrightarrow} \ldots
$$

Clearly one obtains the canonical map

$$
s: H_{n}^{S}(X) \rightarrow H_{n+1}^{S}(\Sigma X)
$$


which is an isomorphism. Hence $\left(H^{S}, s\right)$ is a suspension theory satisfying the suspension axiom. If $(H, s)$ satisfies the exactness or colimit axiom then so does $\left(H^{S}, s\right)$. Clearly for a homology theory we have $(H, s)=\left(H^{S}, s\right)$.

(2.3) Definition. Let $r \geq 0$. A boundary theory $(H, \partial)$ is a sequence of covariant functors, $n \in \mathbb{Z}$,

$$
H_{n}: \text { pair }_{r} \rightarrow \mathbf{A b}
$$

together with a sequence of natural transformations $\partial: H_{n+1}(X, Y) \rightarrow H_{n}(Y)$ with $(X, Y) \in$ pair $_{r}$ and $H_{n}(Y)=H_{n}(Y, *)$, such that $H_{n}(*)=0$ and $H_{n}\left(f_{0}\right)=$ $H_{n}\left(f_{1}\right)$ for homotopic maps $f_{0} \simeq f_{1}$ in pair $r$. Moreover $\partial$-exactness is satisfied, that is

$$
\ldots \rightarrow H_{n+1}(X, Y) \stackrel{\partial}{\longrightarrow} H_{n}(Y) \stackrel{i_{*}}{\longrightarrow} H_{n}(X) \stackrel{j_{*}}{\longrightarrow} H_{n}(X, Y) \stackrel{\partial}{\longrightarrow} \ldots
$$

is exact. Here $i_{*}$ and $j_{*}$ are induced by $i: Y \subset X$ and $j:(X, *) \subset(X, Y)$. We also consider boundary theories with the following additional property.

Excision. For $(X, Y) \in$ pair $_{r}$ and $g: Y \rightarrow Z \in$ space $_{r}$ the adjunction map $\bar{g}$ induces an isomorphism

$$
\bar{g}_{*}: H_{n}(X, Y) \cong H_{n}\left(X \cup_{Y} Z, Z\right)
$$

for all $n \in \mathbb{Z}$.

A boundary theory $(H, \partial)$ is termed a homology theory on pair $r$ if excision is satisfied. Compare [29].

Each boundary theory $(H, \partial)$ yields a suspension theory $(H, s)$ as follows. Let $C X$ be the cone on $X$. Then $\partial: H_{n+1}(C X, X) \cong H_{n}(X)$ is an isomorphism. Hence we obtain the suspension map

$$
s=q_{*} \partial^{-1}: H_{n}(X) \cong H_{n+1}(C X, X) \rightarrow H_{n+1}(\Sigma X)
$$

where $q:(C X, X) \rightarrow(\Sigma X, *)$ is the quotient map. If $(H, \partial)$ is a homology theory then $q_{*} \partial^{-1}$ is an isomorphism since excision implies that $q_{*}: H_{n}(X, Y) \rightarrow$ $H_{n}(X / Y)$ is an isomorphism. This leads to the following well known lemma. For this we observe that suspension theories, resp. boundary theories form categories. Morphisms are the natural transformations compatible with $s$, resp. $\partial$.

(2.5) Lemma. The category of homology theories $(H, \partial)$ on pair $_{r}$ and the category of homology theories $(H, s)$ on space $_{r}$ are equivalent. The equivalence carries $(H, \partial)$ to $\left(H, q^{*} \partial^{-1}\right)$.

These categories actually do not depend on $r$ since the category of homology theories $\left(H^{\prime}, s^{\prime}\right)$ on space $r$ is equivalent to the category of homology theories $(H, s)$ on space. The equivalence carries $\left(H^{\prime}, s^{\prime}\right)$ to $(H, s)$ with $H_{n}(X)=H_{n+r}^{\prime}\left(\Sigma^{r} X\right)$. This shows that a homology theory on space is determined by its restriction to the category space $_{r}$ for arbitrary large $r \geq 0$. Such a statement will not be true for quadratic homology theories below.

(2.6) Example. (A) Let $A$ be an abelian group and let $H_{n}(X, Y ; A)$ be the singular homology of the pair $(X, Y)$ with coefficients in $A$. This is the classical homology theory on pair.

(B) Let $\pi_{n}(X, Y)$ be the relative homotopy group of the pair $(X, Y)$. Then $\left(\pi_{n}, \partial\right)$ is a boundary theory on pair ${ }_{2}$ with $\pi_{n}(X, Y)=0$ for $n \leq 1$. Here we use $(X, Y) \in$ 
pair $_{2}$ in order to obtain abelian groups $\pi_{n}(X, Y), n \in \mathbb{Z}$. The associated stable theory $\left(\pi_{n}^{S}, s\right)$ is the homology theory on space ${ }_{2}$ termed stable homotopy.

The spherical groups of a homology theory $H$ on pair ${ }_{r}$ are the groups $H_{n}\left(S^{r}\right), n \in \mathbb{Z}$, where $S^{r}$ is the $r$-sphere. The suspension isomorphism $H_{n}\left(S^{r}\right) \cong$ $H_{n+k}\left(S^{r+k}\right)$ shows that the spherical groups determine the value of $H$ on all spheres in space $_{r}$. Moreover the following uniqueness-lemma is well known: Let $\phi: H \rightarrow H^{\prime}$ be a natural transformation of homology theories on pair $r$ such that $\phi$ is an isomorphism on spherical groups; that is, $\phi: H_{n}\left(S^{r}\right) \cong H_{n}^{\prime}\left(S^{r}\right)$ for $n \in \mathbb{Z}$. Then $\phi$ is an isomorphism

$$
\phi: H_{n}(X, Y) \cong H_{n}^{\prime}(X, Y)
$$

for all finite $\mathrm{CW}$-pairs $(X, Y) \in$ pair $_{r}$ and $n \in \mathbb{Z}$. Compare for example (2.12) in [28]. A similar uniqueness lemma is also true for the quadratic homology theories below. Moreover we have the following representability theorem of E.H. Brown; compare for example I.3.8 in [28]. Let $H$ be a homology theory on space ${ }_{r}$. Then there exists a spectrum $E$ and a natural isomorphism

$$
H_{n}(X) \cong \pi_{n}(E \wedge X)=H_{n}(X, E), \quad n \in \mathbb{Z},
$$

for all finite $\mathrm{CW}$-spaces $X$ in space $_{r}$.

Next we describe the "partial suspension" and the "cross effect suspension" of a boundary theory. To this end we have to introduce the following notation on cross effects.

(2.9) Notation. Let $\mathbf{G r}$ be the category of groups and let $\mathbf{C}$ be a category with zero object $*$ and assume that sums (coproducts) $X \vee Y$ exist in $\mathbf{C}$. For objects $X, Y \in \mathbf{C}$ one has the unique zero morphism $0: X \rightarrow * \rightarrow Y$. Maps $f: X \rightarrow Z, g: Y \rightarrow Z$ determine $(f, g): X \vee Y \rightarrow Z$. We have the retraction $r_{1}: X \vee Y \rightarrow X$ and $r_{2}: X \vee Y \rightarrow Y$ with $r_{1}=(1,0)$ and $r_{2}=(0,1)$. Given a functor

$$
F: \mathbf{C} \rightarrow \mathbf{G r}
$$

satisfying $F(*)=*$ we define the cross effect

$$
F(X \mid Y)=\operatorname{kernel}\left\{\left(F r_{1}, F r_{2}\right): F(X \vee Y) \rightarrow F(X) \times F(Y)\right\} \text {. }
$$

This yields the functor $F(\mid): \mathbf{C} \times \mathbf{C} \rightarrow \mathbf{G r}$ with induced maps denoted by $(f \mid g)_{*}$. The inclusion $i_{12}: F(X \mid Y) \subset F(X \vee Y)$ is natural in $X$ and $Y$. Moreover we have the natural interchange isomorphism

$$
T: F(X \mid Y) \cong F(Y \mid X)
$$

induced by $T: X \vee Y=Y \vee X$ with $T=\left(i_{2}, i_{1}\right)$. Let $F(X \vee Y)_{2}$ be the kernel of $F r_{2}: F(X \vee Y) \rightarrow F(Y)$. Then we have the split short exact sequence of groups

$$
0 \rightarrow F(X \mid Y) \stackrel{i_{12}}{\longrightarrow} F(X \vee Y)_{2} \stackrel{F r_{1}}{\longrightarrow} F(X) \rightarrow 0
$$

If $F$ is a functor $\mathbf{C} \rightarrow \mathbf{A b}$ we thus have natural isomorphisms

$$
\begin{gathered}
\left(F i_{1}, i_{12}\right): F(X) \oplus F(X \mid Y)=F(X \vee Y)_{2}, \\
\left(F i_{1}, F i_{2}, i_{12}\right): F(X) \oplus F(Y) \oplus F(X \mid Y)=F(X \vee Y)
\end{gathered}
$$

which we use as identifications. The functor $F: \mathbf{C} \rightarrow \mathbf{G r}$ is linear if $F(*)=0$ and $F(X \mid Y)=0$ for all $X, Y \in \mathbf{C}$; that is

$$
\left(F r_{1}, F r_{2}\right): F(X \vee Y) \cong F(X) \times F(Y)
$$


is an isomorphism. The functor $F: \mathbf{C} \rightarrow \mathbf{G r}$ is quadratic if $F(*)=0$ and the cross effect $F(X \mid Y)$ as a bifunctor is linear in each variable $X$ and $Y$. If $F$ is linear then the group $F(X)$ is abelian and if $F$ is quadratic then the group $F(X)$ has nilpotency degree 2. Moreover for a quadratic functor the subgroup $F(X \mid Y)$ is central in $F(X \vee Y)$. Compare [10].

Now let $(H, \partial)$ be a boundary theory as in (2.3). Then one gets the following exact sequences from which we derive the partial suspension $E$. Let $C Y$ be the cone on $Y$ so that $H_{n}(C Y \vee X)=H_{n}(X)$. This yields the short exact sequence

$$
0 \rightarrow H_{n+1}(C Y \vee X, Y \vee X) \stackrel{\partial}{\longrightarrow} H_{n}(Y \vee X) \stackrel{r_{2}}{\longrightarrow} H_{n}(X) \rightarrow 0
$$

and hence the isomorphism

$$
\partial_{0}: H_{n+1}(C Y \vee X, Y \vee X) \cong H_{n}(Y \vee X)_{2} .
$$

Moreover one gets the short exact sequence

$$
0 \rightarrow H_{n+1}(Y) \rightarrow H_{n+1}(\Sigma X \vee Y) \stackrel{j_{*}}{\longrightarrow} H_{n+1}(\Sigma X \vee Y, Y) \rightarrow 0
$$

which shows that $j_{*}$ induces the isomorphism

$$
j_{0}: H_{n+1}(\Sigma X \vee Y)_{2} \cong H_{n+1}(\Sigma X \vee Y, Y)
$$

Dividing out $X$ yields the quotient map $q \vee 1:(C X \vee Y, X \vee Y) \rightarrow(\Sigma X \vee Y, Y)$. Now the partial suspension $E$ is the composition

$$
E=j_{0}^{-1}(g \vee 1)_{*} \partial_{0}^{-1}: H_{n}(X \vee Y)_{2} \rightarrow H_{n+1}(\Sigma X \vee Y)_{2}
$$

Compare $[2,3]$. If $Y=*$ this is the suspension in (2.4). Moreover the partial suspension restricts to cross effects yielding the cross effect suspension

$$
s: H_{n}(X \mid Y) \rightarrow H_{n+1}(\Sigma X \mid Y)
$$

such that via (2.9) (4) the partial suspension is the composite

$$
\begin{array}{ccc}
H_{n}(X \vee Y)_{2} & \multicolumn{1}{l}{} & H_{n+1}(\Sigma X \vee Y)_{2} \\
\| & \\
H_{n}(X) \oplus H_{n}(X \mid Y) \stackrel{s \oplus s}{\longrightarrow} H_{n+1}(\Sigma X) \oplus H_{n+1}(\Sigma X \mid Y)
\end{array}
$$

Hence for each $Y$ the pair $\left(H_{n}(-\mid Y), s\right)$ is a suspension theory. Using the interchange map $T$ in $(2.9)(2)$ also $\left(H_{n}(X \mid-), \bar{s}\right)$ is a suspension theory where $\bar{s}$ is the composite

$$
\bar{s}=T s T: H_{n}(X \mid Y) \cong H_{n}(Y \mid X) \rightarrow H_{n+1}(\Sigma Y \mid X) \cong H_{n+1}(X \mid \Sigma Y) .
$$

The sum or coproduct in the category space $_{r}$ is obtained by the one point union $X \vee Y$ of spaces.

(2.13) Lemma. Let $H$ be a homology theory on pair ${ }_{r}$. Then $H_{n}:$ space $_{r} \rightarrow \mathbf{A b}$ is linear, that is $H_{n}(X \mid Y)=0$ and $H_{n}(X \vee Y)=H_{n}(X) \oplus H_{n}(Y)$.

Proof. For the CW-pair $(X \vee Y, Y) \in$ pair $_{r}$ we have by exactness the split short exact sequence

$$
0 \rightarrow H_{n}(Y) \rightarrow H_{n}(X \vee Y) \rightarrow H_{n}(X \vee Y, Y) \rightarrow 0
$$

where $H_{n}\left(i_{2}\right)$ is injective since we have the retraction $H_{n}\left(r_{2}\right)$. Moreover excision shows that $H_{n}(X) \rightarrow H_{n}(X \vee Y, Y)$ is an isomorphism. 
(2.14) Lemma. Let $(H, \partial)$ be a boundary theory on pair ${ }_{r}$. Then the suspension $s$ in (2.4) makes the following diagram commute

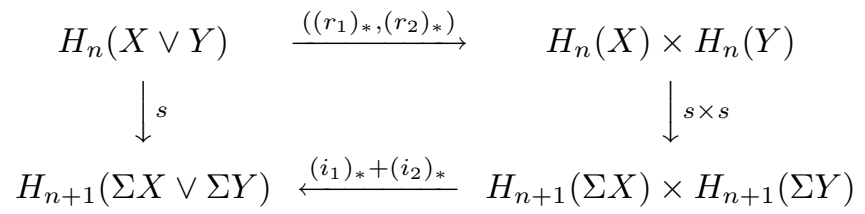

This implies that the composition

$$
H_{n}(X \mid Y) \stackrel{i_{12}}{\longrightarrow} H_{n}(X \vee Y) \stackrel{s}{\longrightarrow} H_{n+1}(\Sigma X \vee \Sigma Y)
$$

is always trivial, $s i_{12}=0$.

Proof of (2.14). Let $\pi: C X \rightarrow \Sigma X$ be the quotient map. Then

$$
C(X \vee Y)=C(X) \vee C(Y) \stackrel{\pi_{X} \vee 1}{\longrightarrow} \Sigma X \vee C Y \stackrel{1 \vee \pi_{Y}}{\longrightarrow} \Sigma X \vee \Sigma Y=\Sigma(X \vee Y)
$$

is the quotient map $\pi_{X \vee Y}$. Hence we get the following diagram in which the row is split short exact.

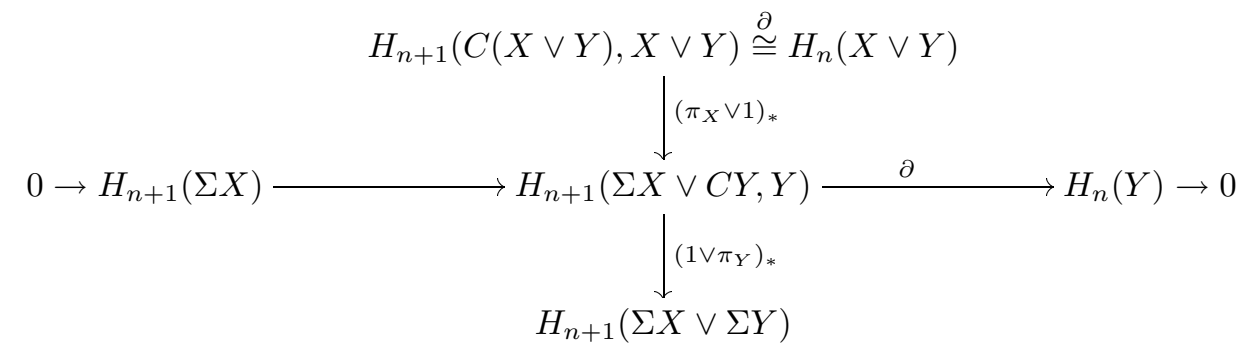

Here we have the isomorphism $\left(i_{1}\right)_{*}+\left(i_{2}\right)_{*}$

$$
H_{n+1}(\Sigma X) \oplus H_{n+1}(C Y, Y) \stackrel{\cong}{\longrightarrow} H_{n+1}(\Sigma X \vee C Y, Y)
$$

by the exactness of the row. This yields the result.

\section{Quadratic homology}

In this section we introduce new axioms of a quadratic homology theory. These axioms are satisfied by various examples which we describe in the following sections. We deal with some consequences of the axioms, in particular we obtain the long EHP-sequence of quadratic homology and we prove a uniqueness lemma.

(3.1) Notation. Let $F: \mathbf{C} \rightarrow \mathbf{G r}$ be a functor where $\mathbf{C}$ is a category with zero object $*$ and sums and let $F(*)=0$. We obtain the natural homomorphism

$$
P: F(X \mid X) \stackrel{i_{12}}{\complement} F(X \vee X) \stackrel{(1,1)_{*}}{\longrightarrow} F(X)
$$

for $X \in \mathbf{C}$. Moreover if $X$ is a cogroup in $\mathbf{C}$ with structure maps $\mu: X \rightarrow X \vee X$ and $\nu: X \rightarrow X$ satisfying the usual identities we define the function $H$, with

$$
F(X) \stackrel{H}{\longrightarrow} F(X \mid X) \stackrel{i_{12}}{\complement} F(X \vee X)
$$


by $i_{12} H(a)=F(\mu)(a)-F\left(i_{2}\right)(a)-F\left(i_{1}\right)(a)$ where $i_{1}, i_{2}$ are the inclusions of $X$ in $X \vee X$. Clearly $H$ is natural with respect to maps between cogroups. We write $F\{X\}$ for the pair of functions

$$
F\{X\}=(F(X) \stackrel{H}{\longrightarrow} F(X \mid X) \stackrel{P}{\longrightarrow} F(X)) .
$$

It is shown in $3.6[10]$ that $F\{X\}$ is a square group (see (6.3) below) if $F$ is a quadratic functor. Clearly $H$ is a homomorphism for a functor $F: \mathbf{C} \rightarrow \mathbf{A b}$. If $\mathbf{C}$ is an additive category then a quadratic functor $F: \mathbf{C} \rightarrow \mathbf{A b}$ yields for $X \in \mathbf{C}$ a pair of homomorphisms $F\{X\}=(H, P)$ as above with $H P H=2 H$ and $P H P=2 P$; that is $F\{X\}$ is a quadratic $\mathbb{Z}$-module; compare [5].

(3.2) Definition. Let $r \geq 0$ and consider a boundary theory $(Q, \partial)$ on pair $r$ with the following properties (i) and (ii).

(i) The stable theory $Q^{S}$ associated to $Q$ via the suspension (2.4) is a homology theory.

(ii) For each $Y \in$ space $_{r}$ the suspension theory of cross effects $(Q(-\mid Y), s)$ in (2.11) is a homology theory.

A quadratic homology theory $(Q, \partial, \delta)$ is a boundary theory $(Q, \partial)$ which satisfies (i) and (ii) together with a sequence of homomorphisms, $n \in \mathbb{Z}$,

$$
\delta: Q_{n+1}\left(C A \cup_{A} X, X\right) \rightarrow Q_{n-1}(A \mid A)
$$

which are defined for all pairs $(X, A)$ in pair $r$. The homomorphisms $\delta$ might look obscure to the reader; yet the cubical diagram in the proof of (4.12) below (defined for any quadratic homotopy functor) yields canonically such homomorphisms $\delta$; see (4.12) (5). The homomorphisms $\delta$ are natural in $(X, A)$, that is: A pair map $f:(X, A) \rightarrow(Y, B) \in$ pair $_{r}$ induces a commutative diagram

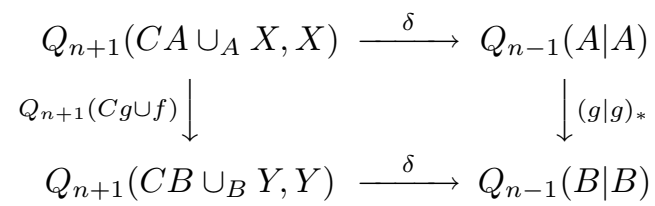

where $g: A \rightarrow B$ is the restriction of $f$. Moreover the following property (iii) is satisfied.

(iii) For each pair $(X, A)$, the sequence

$$
\begin{aligned}
\ldots \stackrel{\delta}{\longrightarrow} Q_{n}(A \mid A) \stackrel{j_{\sharp}}{\longrightarrow} Q_{n+1}(C A \vee X, A \vee X) \\
\stackrel{i_{\sharp}}{\longrightarrow} Q_{n+1}\left(C A \cup_{A} X, X\right) \stackrel{\delta}{\longrightarrow} Q_{n-1}(A \mid A) \rightarrow \ldots
\end{aligned}
$$

is exact. Here the inclusion $i: A \rightarrow X$ yields the map $(i, 1): A \vee X \rightarrow X$ which defines the adjunction map $\overline{(i, 1)}:(C A \vee X, A \vee X) \rightarrow\left(C A \cup_{A} X, X\right)$ which induces $i_{\sharp}=\overline{(i, 1)}_{*}$ in the sequence. Moreover $j_{\sharp}$ in the sequence is the composition

$$
\begin{aligned}
j_{\sharp} & =\partial_{0}^{-1}\left(P,-(1 \mid i)_{*}\right): Q_{n}(A \mid A) \rightarrow Q_{n}(A) \oplus Q_{n}(A \mid X) \\
& =Q_{n}(A \vee X)_{2} \cong Q_{n+1}(C A \vee X, A \vee X) .
\end{aligned}
$$

Here $P$ is the natural map in (3.1) and $-(1 \mid i)_{*}$ is the negative of the induced homomorphism $(1 \mid i)_{*}=Q_{n}(1 \mid i)$. We call the long exact sequence $\left(j_{\sharp}, i_{\sharp}, \delta\right)$ above the quadratic excision sequence. 
It follows from (ii) above and (2.13) that all of the functors

$$
Q_{n}: \text { space }_{r} \rightarrow \underline{\underline{A b}}
$$

are quadratic. Moreover one can check that a quadratic homology theory $(Q, \partial, \delta)$ for which these functors $Q_{n}$ are linear is the same as a homology theory on pair . $^{\text {. }}$ This follows from (i) and (3.5) below.

Remark. Clearly axioms as described in (3.2) are obtained by choosing basic properties of the examples of quadratic homology theories which we consider below. We have chosen the axioms in such a way that they are very close to the familiar concepts of a "boundary theory" (2.3), "cross effect" (3.1) and "EHP-sequence" (3.5) and such that the uniqueness lemma (3.8) for these axioms is satisfied. A different choice of axioms could be obtained by transforming Goodwillie's definition of 2excisive homotopy functors; this however would involve functors defined on triads $(X ; A, B)$ of spaces. In view of the theorem in Appendix $\mathrm{A}$ the use of such triad groups is not necessary. It remains open to find a set of axioms for a quadratic homology theory which implies the quadratic analogue of Brown's representability theorem; see the remark following (4.7). In any case the basic properties described in (3.2) should be derivable from any good set of axioms describing "quadratic homology theories".

We now derive from the axioms of a quadratic homology theory some consequences. First we observe that the following diagram commutes.

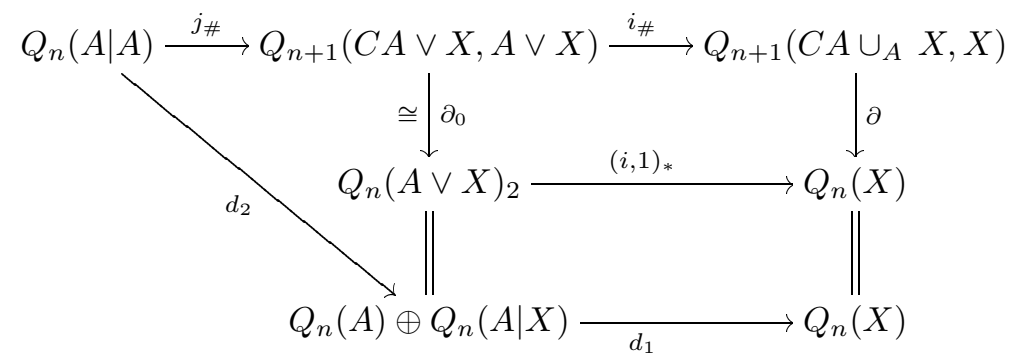

where $d_{2}=\left(P,-(1 \mid i)_{*}\right)$ and $d_{1}=\left(i_{*}, P(i \mid 1)_{*}\right)$. Here $d_{1} d_{2}=0$ since $P$ is natural; in fact,

$$
d_{1} d_{2}=i_{*} P-P(i \mid 1)_{*}(1 \mid i)_{*}=i_{*} P-P(i \mid i)_{*}=0 .
$$

Clearly $d_{1} d_{2}=0$ also follows from $i_{\sharp} j_{\sharp}=0$ and the commutativity of the diagram. Using (3.3) we can equivalently describe the excision exact sequence in (3.2) (iii) by the exact sequence in the row of the commutative diagram:

$$
\cdots \stackrel{\delta}{\longrightarrow} Q_{n}(A \mid A) \stackrel{d_{2}}{\longrightarrow} Q_{n}(A) \oplus Q_{n}(\underbrace{\stackrel{\bar{d}_{1}}{\longrightarrow}}_{d_{1}} Q_{n+1}\left(C A \cup_{Q_{n}(X)}^{\rfloor_{\partial}}\right.
$$

Here we set $\bar{d}_{1}=i_{\sharp} \partial_{0}^{-1}$ by (3.4). This is similar to the diagram for metastable homotopy groups in 2.4 of [4]. As a consequence of (3.4) we obtain a long exact sequence which resembles the classical EHP-sequence of James [23]. 
(3.5) Proposition. Let $Q$ be a quadratic homology theory on pair $_{r}, r \geq 0$. Then one has the following long exact sequence which is natural in $A \in$ space $_{r}$.

$$
\ldots \rightarrow Q_{n}(A \mid A) \stackrel{P}{\longrightarrow} Q_{n}(A) \stackrel{s}{\longrightarrow} Q_{n+1}(\Sigma A) \stackrel{H^{\prime}}{\longrightarrow} Q_{n-1}(A \mid A) \stackrel{P}{\longrightarrow} Q_{n-1}(A) \rightarrow \ldots
$$

This exact sequence is deduced purely algebraically from the axioms (3.2). Given a quadratic homotopy functor $D$ we always obtain the EHP sequence since (4.7) below holds. The EHP sequence for the quadratic homotopy functor $D$ can also be deduced from Goodwillie's classification of quadratic homotopy functors. In fact $D(X)$ is given as the fiber of a natural map $\Omega^{\infty}\left(D_{1} \wedge X\right) \rightarrow \Omega^{\infty}\left(D_{2} \wedge X \wedge X / \Sigma_{2}\right)$ where $D_{1}$ and $D_{2}$ are spectra; see (8.2). As pointed out by the referee applying the natural map $D(X) \rightarrow \Omega D(\Sigma X)$ to this fibration yields as well the EHP-sequence for $D$.

Proof. We consider (3.4) in case $X=C A$ is the cone on $A$. Then the homotopy axiom shows $Q_{n}(A \mid C A)=0$ and $Q_{n}(C A)=0$. Hence we obtain the isomorphism

$$
i_{*}: Q_{n+1}\left(C A \cup_{A} C A\right) \cong Q_{n+1}\left(C A \cup_{A} C A, C A\right)
$$

where $\Sigma A=C A \cup \cup_{A} C A$. Thus (3.4) yields the commutative diagram

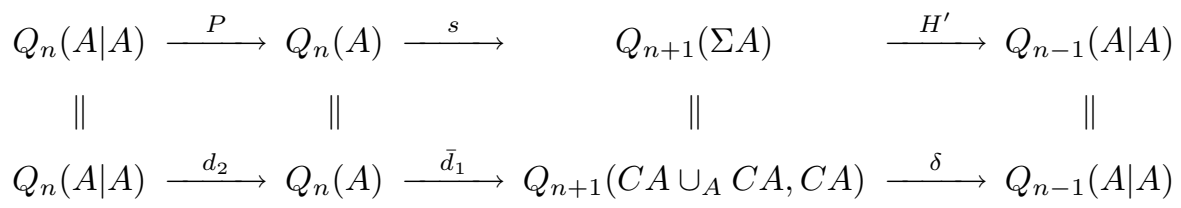

which defines the exact sequence in (3.5).

Given a natural exact sequence as in (3.5) one obtains the associated sequence of cross effects which is also exact. Since $s i_{12}=0$ by (2.14) this yields the following commutative diagram with exact rows.

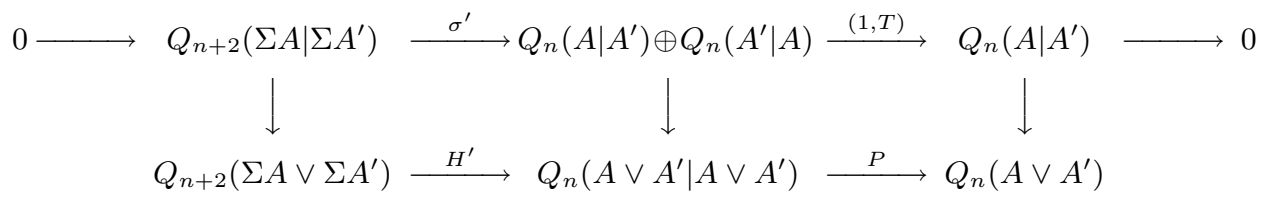

Here the vertical arrows are the inclusions $i_{12}$. This shows that $\sigma^{\prime}$ induces the isomorphism

$$
\sigma: Q_{n+2}\left(\Sigma A \mid \Sigma A^{\prime}\right) \cong Q_{n}\left(A \mid A^{\prime}\right)
$$

where $\sigma=\left(p r_{1}\right) \sigma^{\prime}$ is the composition of $\sigma^{\prime}$ and the projection $p r_{1}$. Clearly $\sigma$ is again natural in $A$ and $A^{\prime}$.

Remark. Using the suspension isomorphism $\bar{s} s$ in (3.2) (ii) and (2.11), (2.12) we obtain a further natural isomorphism

$$
\bar{s} s: Q_{n}\left(A \mid A^{\prime}\right) \cong Q_{n+2}\left(\Sigma A \mid \Sigma A^{\prime}\right) .
$$

The relationship between $\sigma$ and $\bar{s} s$ remains unsettled. 
(3.7) Proposition. Let $A$ be a cogroup in space $_{r} / \simeq$, for example let $A$ be a suspension. Then the operator $H^{\prime}$ in (3.5) and $H$ in (3.1) yield the following commutative diagram

$$
\begin{gathered}
Q_{n+1}(\Sigma A) \\
H \swarrow \quad \searrow H^{\prime} \\
Q_{n+1}(\Sigma A \mid \Sigma A) \underset{\sigma}{\stackrel{\cong}{\leftrightarrows}} Q_{n-1}(A \mid A)
\end{gathered}
$$

where $\sigma$ is the isomorphism in (3.6).

Proof. Let $\mu: A \rightarrow A \vee A^{\prime}$ be the comultiplication of $A$ with $A=A^{\prime}$. Then $\Sigma \mu$ is the comultiplication of the suspension $\Sigma A$. Now the naturality of $H^{\prime}$ in (3.5) shows that the following diagram commutes

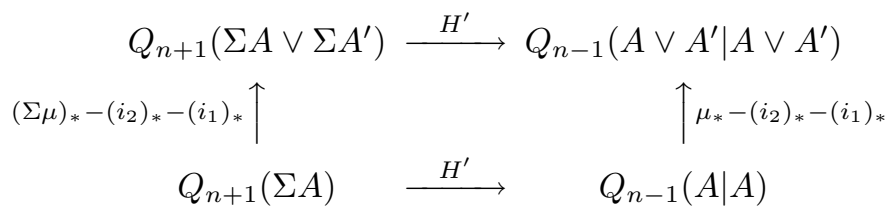

Here the vertical arrows map to the cross effects; in fact the left hand side is $i_{12} H$ by definition of $H$ and the right hand side is $i_{12}(1, T)$ by the bilinearity of the cross effect. This implies by the definition of $\sigma^{\prime}$ above that $\sigma^{\prime} H=(1, T) H^{\prime}$ and hence $\sigma H=H^{\prime}$.

For the metastable range of homotopy groups one has a diagram as in (3.7) where $\sigma$ is actually the double suspension up to sign; compare A.6.8 in [7] and example (2.6) (B) above.

The next lemma justifies our choice of axioms of a quadratic homology theory. It is the quadratic analogue of the uniqueness lemma in (2.7) above. The spherical groups of a quadratic homology theory $Q$ on pair $r$ are the groups $Q_{n}\left(S^{r+k}\right)$ and $Q_{n}\left(S^{r} \mid S^{r}\right), n \in \mathbb{Z}, k \geq 0$. Now the following uniqueness lemma holds.

(3.8) Lemma. Let $\phi: Q \rightarrow Q^{\prime}$ be a natural transformation of quadratic homology theories on pair $_{r}$ compatible with $\partial$ and $\delta$. Moreover assume $\phi$ is an isomorphism on spherical groups. Then $\phi$ is an isomorphism

$$
\phi: Q_{n}(X, Y) \cong Q_{n}^{\prime}(X, Y)
$$

for all finite $C W$-pairs $(X, Y) \in$ pair $_{r}$ and $n \in \mathbb{Z}$.

Proof. Since $Q_{n}(\mid)$ is a homology theory in each variable we see that $\phi$ induces an isomorphism $Q_{n}(X \mid Z) \cong Q_{n}^{\prime}(X \mid Z)$ for all finite $X, Z$. Compare (2.7). This shows that for $k \geq 0$ one gets the isomorphism $\phi: Q_{n}(X) \cong Q_{n}^{\prime}(X)$ for all spaces $X$ which are finite one point unions of spheres $S^{r+k}$. By exactness it suffices to show that $\phi$ induces an isomorphism $\phi_{X}: Q_{n}(X) \cong Q_{n}^{\prime}(X)$ for all finite $X$ in space ${ }_{r}$. We proceed by induction on the dimension of $X$. If $\operatorname{dim}(X)=r$ then $X$ is a finite one point union of spheres $S^{r}$. Now assume $\phi_{X}$ is an isomorphism for all finite $X$ with $\operatorname{dim}(X)<r+k$ and let $\operatorname{dim}(Y)=r+k$. Then we may assume that $Y=C A \cup_{A} X$ where $(X, A)$ is a pair with $\operatorname{dim}(X)<r+k$ and $A$ is a finite one point union of 
spheres $S^{r+k-1}$. Hence $\phi_{X}$ and $\phi_{A}$ are isomorphisms and therefore the quadratic excision sequence shows that also

$$
\phi: Q_{n+1}(Y, X) \cong Q_{n+1}^{\prime}(Y, X)
$$

is an isomorphism. Hence $\partial$-exactness shows that $\phi_{Y}$ is an isomorphism since $\phi_{X}$ is one.

Now let $(Q, \partial, \delta)$ be a quadratic homology theory on space $r$ and let $n \geq r, m \in$ $\mathbb{Z}$. By (3.1) we obtain the quadratic $\mathbb{Z}$-module

$$
Q_{m}\left\{S^{n}\right\}=\left(Q_{m}\left(S^{n}\right) \stackrel{H}{\longrightarrow} Q_{m}\left(S^{n} \mid S^{n}\right) \stackrel{P}{\longrightarrow} Q_{m}\left(S^{n}\right)\right)
$$

which describes the canonical quadratic structure of the spherical groups above. We now recall the following notation concerning quadratic $\mathbb{Z}$-modules; compare [5].

(3.10) Definition. Let $M=\left(M_{e} \stackrel{H}{\longrightarrow} M_{e e} \stackrel{P}{\longrightarrow} M_{e}\right)$ be a quadratic $\mathbb{Z}$-module, i.e. a pair of homomorphisms $H$ and $P$ with $H P H=2 H$ and $P H P=2 P$. Then $M$ induces functors $\mathbf{A b} \rightarrow \mathbf{A b}$ which carry $A$ to $A \otimes M, A *^{\prime} M$ and $A *^{\prime \prime} M$ respectively. Here $A \otimes M$ is the abelian group with generators $a \otimes m,[a, b] \otimes n$ for $a, b \in A, m \in M_{e}, n \in M_{e e}$ and relations

$$
\begin{aligned}
(a+b) \otimes m & =a \otimes m+b \otimes m+[a, b] \otimes H m, \\
{[a, a] \otimes n } & =a \otimes P(n),
\end{aligned}
$$

where $a \otimes m$ is linear in $m$ and $[a, b] \otimes n$ is linear in $a, b$ and $n$. Now let

$$
0 \rightarrow A_{1} \stackrel{d}{\longrightarrow} A_{0} \stackrel{q}{\longrightarrow} A \rightarrow 0
$$

be a short exact sequence in $\mathbf{A b}$ where $A_{0}$ is free abelian. Then we obtain homomorphisms

$$
A_{1} \otimes A_{1} \otimes M_{e e} \stackrel{d_{2}}{\longrightarrow} A_{1} \otimes M \oplus A_{1} \otimes A_{0} \otimes M_{e e} \stackrel{d_{1}}{\longrightarrow} A_{0} \otimes M
$$

with $d_{1} d_{2}=0$ as follows:

$$
\begin{aligned}
d_{1}(a \otimes m) & =(d a) \otimes m, \\
d_{1}\left(\left[a, a^{\prime}\right] \otimes n\right) & =\left[d a, a^{\prime}\right] \otimes n, \\
d_{1}(a \otimes b \otimes n) & =[d a, b] \otimes n, \\
d_{2}\left(a \otimes a^{\prime} \otimes n\right) & =-a \otimes d a^{\prime} \otimes n+\left[a, d a^{\prime}\right] \otimes n
\end{aligned}
$$

for $a, a^{\prime} \in A_{1}, b \in A_{0}, m \in M_{e}, n \in M_{e e}$. One can check that $\operatorname{cok}\left(d_{1}\right)=A \otimes M$. Moreover we set

$$
\begin{aligned}
& A *^{\prime} M=\operatorname{ker}\left(d_{1}\right) / \operatorname{im}\left(d_{2}\right), \\
& A *^{\prime \prime} M=\operatorname{ker}\left(d_{2}\right) .
\end{aligned}
$$

These are the derived functors of the functor $\mathbf{A b} \rightarrow \mathbf{A b}$ which carries $A$ to $A \otimes M$. An abelian group $N \in \mathbf{A b}$ yields the quadratic $\mathbb{Z}$-module $N=(N \rightarrow 0 \rightarrow N)$ with $N_{e e}=0$. In this case $A \otimes N$ is the usual tensor product and $A * N=A *^{\prime} N$ is the usual torsion product. Clearly $A *^{\prime \prime} N=0$.

We now consider the quadratic homology of a Moore space $M(A, n)$. A Moore space $M(A, n), n \geq 2$, is a simply connected CW-space $X$ with homology $H_{n}(X, \mathbb{Z})$ $=A$ and $\tilde{H}_{i}(X, \mathbb{Z})=0$ otherwise. If $A$ is a free abelian group then $M(A, n)$ is a one point union of spheres $S^{n}$, in particular $M(\mathbb{Z}, n)=S^{n}$. Each homomorphism 
$\varphi: A \rightarrow B$ in $\mathbf{A b}$ admits a realization $\bar{\varphi}: M(A, n) \rightarrow M(B, n)$ with $H_{n}(\bar{\varphi})=$ $\varphi$. Here $\bar{\varphi}$ is unique up to homotopy if $A$ is free abelian. There is the natural homomorphism

$$
\lambda: A \otimes Q_{m}\left\{S^{n}\right\} \rightarrow Q_{m}(M(A, n))
$$

defined as follows. For $a, b \in A=\pi_{n} M(A, n), u \in Q_{m}\left(S^{n}\right), v \in Q_{m}\left(S^{n} \mid S^{n}\right)$ we set

$$
\begin{aligned}
\lambda(a \otimes u) & =Q_{m}(a)(u), \\
\lambda([a, b] \otimes v) & =P Q_{m}(a \mid b)(v) .
\end{aligned}
$$

Let ${ }_{\lambda} Q_{m}(M(A, n))$ be the cokernel of $\lambda$.

(3.12) Proposition. Assume that the quadratic homology $(Q, \partial, \delta)$ satisfies the colimit axiom. Then $\lambda$ above is an isomorphism in case $A$ is a free abelian group. Moreover for a general abelian group $A \in \mathbf{A b}$ there is the natural exact sequence $(n \geq 2, m \in \mathbb{Z})$ :

$$
\begin{aligned}
& 0 \rightarrow A *^{\prime} Q_{m}\left\{S^{n}\right\} \stackrel{e}{\longrightarrow} Q_{m+1}(M(A, n)) \stackrel{h}{\longrightarrow} A *^{\prime \prime} Q_{m-1}\left\{S^{n}\right\} \\
& \stackrel{\partial}{\longrightarrow} A \otimes Q_{m}\left\{S^{n}\right\} \stackrel{\lambda}{\longrightarrow} Q_{m}(M(A, n)) \rightarrow_{\lambda} Q_{m}(M(A, n)) \rightarrow 0 .
\end{aligned}
$$

This result generalizes 9.3 in [5]. If $Q$ does not satisfy the colimit axiom then Proposition (3.12) is still true for finitely generated abelian groups.

Proof. If $A$ is free abelian we see by (3.6) [5] or (6.4) below that $\lambda$ is an isomorphism. Now let $A \in \mathbf{A b}$ and let

$$
d: X=M\left(A_{1}, n\right) \rightarrow Y=M\left(A_{0}, n\right)
$$

be a map which realizes $d$ in (3.10). Then the Moore space $M(A, n)$ is the mapping cone $M(A, n)=C X \cup_{d} Y$. Therefore we get by (3.4) the following commutative diagram in which the column and the row are exact.

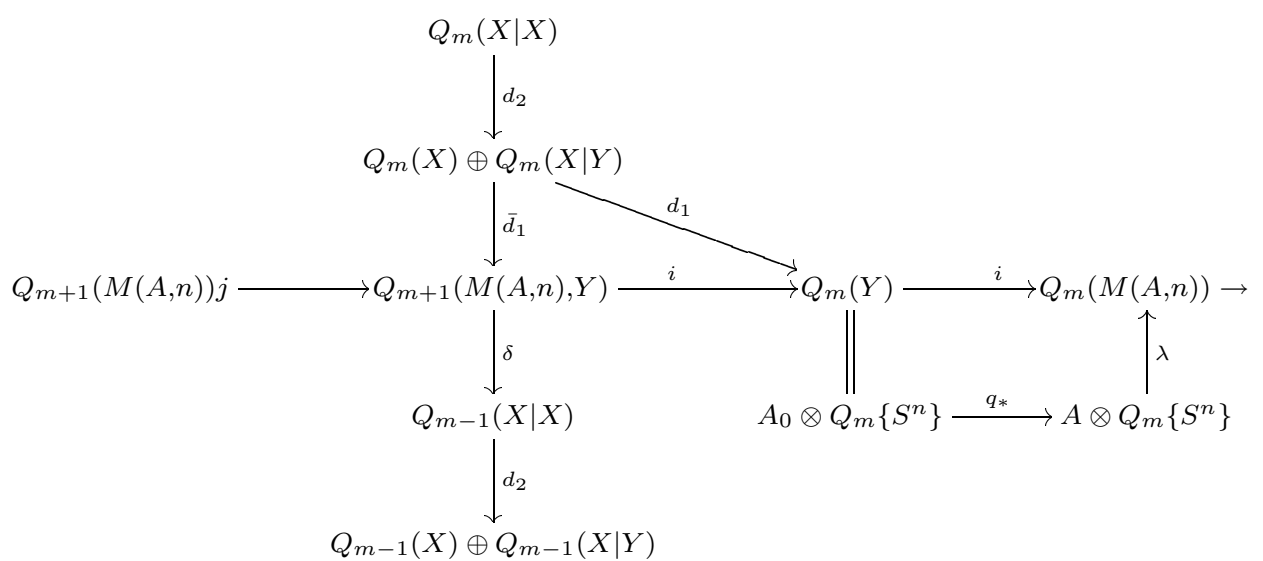

The map $q_{*}$ is surjective and the kernel of $q_{*}$ is the image of $d_{1}$. Moreover using the definition of $d_{1}, d_{2}$ in (3.4) and (3.10) we get for $d_{1}, d_{2}$ in the diagram

$$
\begin{aligned}
\operatorname{ker}\left(d_{1}\right) / \operatorname{im}\left(d_{2}\right) & =A *^{\prime} Q_{m}\left\{S^{n}\right\}, \\
\operatorname{ker}\left(d_{2}\right) & =A *^{\prime \prime} Q_{m}\left\{S^{n}\right\} .
\end{aligned}
$$

We now define the operators in (3.12) as follows. The inclusion $e$ is induced by $\bar{d}_{1}$ where $\operatorname{cok}(i)=\operatorname{cok}(\lambda)=\operatorname{im}(j)$. The map $h$ is the restriction of $\delta$. The map $\partial$ 
in the proposition is induced by $\partial(\delta)^{-1}$ and $e$ and $h$ in the proposition are derived from $i$ and $j$ in the row of the diagram.

\section{Homotopy FunCTORS}

Following Goodwillie $[17,18,19,20,21]$ we consider for $r \geq 0$ functors $D$ of the form

$$
\begin{aligned}
& D: \text { space }_{r} \rightarrow \text { space } \quad \text { or } \\
& D: \text { space }_{r} \rightarrow \text { spectra }
\end{aligned}
$$

We say that $D$ is a homotopy functor if $D$ carries weak equivalences to weak equivalences and preserves filtered colimits up to homotopy. That is, for each sequence of cofibrations $X_{0} \longmapsto X_{1} \longmapsto \ldots$ in space ${ }_{r}$ the induced map

$$
\operatorname{hocolim}\left(D X_{i}\right) \rightarrow D\left(\operatorname{colim} X_{i}\right)
$$

is a weak equivalence. Here hocolim is the homotopy colimit. We say that $D$ is reduced if $* \rightarrow D(*)$ is a weak equivalence. We shall consider various examples of such homotopy functors below.

(4.2) Definition. Let $D$ be a homotopy functor as in (4.1). We define the $D$ homology of a space $X \in$ space $_{r}$ by the homotopy group

$$
H_{n}(X ; D)=\pi_{n}(D(X)) .
$$

Moreover the relative D-homology is the relative homotopy group

$$
H_{n+1}(X, Y ; D)=\pi_{n+1}(D(X), D(Y))
$$

for $(X, Y) \in$ pair $_{r}$. This yields the natural boundary map

$$
\partial: H_{n+1}(X, Y ; D) \rightarrow H_{n}(Y ; D) .
$$

If $D$ maps to spectra then all such homotopy groups are well defined abelian groups for $n \in \mathbb{Z}$. If $D$ maps to space then these groups are abelian only for $n \geq 2$. For $n=1$, resp. $n=0$, the $D$-homology $H_{n}(X, D)$ and $H_{n+1}(X, Y ; D)$ is a group, resp. a pointed set. If $D$ maps to space ${ }_{1}$ we set $H_{n}(X, D)=H_{n}(X, Y ; D)=0$ for $n \leq 0$. If $D$ is a reduced homotopy functor we obtain as in (2.4) the suspension

$$
s=q_{*} \partial^{-1}: H_{n}(X ; D) \rightarrow H_{n+1}(\Sigma X ; D)
$$

which defines the stable theory $H_{n}^{S}(X ; D)$ of stable homology groups with coefficients in $D$.

(4.3) Remark. We have for a reduced homotopy functor $D$ the linearization $D^{\text {lin }}$ which is the homotopy functor obtained by the homotopy colimit of

$$
D(X) \rightarrow \Omega D(\Sigma X) \rightarrow \Omega^{2} D\left(\Sigma^{2} X\right) \rightarrow \ldots
$$

We clearly have the natural isomorphism

$$
H_{n}^{S}(X, D)=H_{n}\left(X, D^{\text {lin }}\right) .
$$

Goodwillie [17] showed that $H_{n}^{S}(X ; D)$ is a homology theory if $D$ is approximately 1-excisive. This is also true if $D$ is $n$-excisive for $n \geq 1$ as follows from 3.2.4 in [20].

The following lemma is an easy consequence of the definitions. 
(4.4) Lemma. Let $D$ be a reduced homotopy functor which maps to space ${ }_{2}$ or spectra. Then D-homology is a boundary theory which satisfies the colimit axiom in (2.1).

Proof. The $\partial$-exact sequence is otained by (2.6) (B). Moreover homotopic maps $f \simeq g$ induce $f_{*}=g_{*}$ in $D$-homology since the projection $I(X) \rightarrow X$ of the cylinder is a homotopy equivalence and hence a weak equivalence.

By an $n$-cube of spaces (or spectra) we will mean the following. Let $\mathbf{P}(n)$ be the category whose objects are the subsets $K$ of $\{1,2, \ldots, n\}$ and whose morphisms are the inclusion maps among the subsets. An $n$-cube $\underline{X}$ in a category $\mathbf{C}$ is a covariant functor $\underline{X}: \mathbf{P}(n) \rightarrow \mathbf{C}$. Goodwillie defines and uses particular $n$-cubes in space or spectra, namely Cartesian and co-Cartesian $n$-cubes. Let "holim" be the homotopy inverse limit and "hocolim" be the homotopy colimit as defined in Bousfield-Kan [12]. Let $P^{\prime}(n)$, resp. $P^{\prime \prime}(n)$, be the full subcategory of $\mathbf{P}(n)$ consisting of all $K$ with $K \neq \phi$, resp. $K \neq\{1, \ldots, n\}$, and let $\underline{X}^{\prime}$ and $\underline{X}^{\prime \prime}$ be the restrictions of $\underline{X}$ to $\mathbf{P}^{\prime}(n)$, resp. $\mathbf{P}^{\prime \prime}(n)$. There are maps

$$
\begin{aligned}
& a(\underline{X}): \underline{X}(\phi)=\lim (\underline{X}) \simeq \operatorname{holim}(\underline{X}) \rightarrow \operatorname{holim}\left(X^{\prime}\right), \\
& b(\underline{X}): \operatorname{hocolim}\left(\underline{X}^{\prime \prime}\right) \rightarrow \operatorname{hocolim}(\underline{X}) \simeq \operatorname{colim}(\underline{X})=\underline{X}(\{1, \ldots, n\}) .
\end{aligned}
$$

Now $\underline{X}$ is Cartesian, resp. co-Cartesian, if $a(\underline{X})$, resp. $b(\underline{X})$, is a weak equivalence. An $n$-cube is strongly co-Cartesian if each of its 2 -faces is co-Cartesian. A Cartesian 2-cube is also called a homotopy pull back and a co-Cartesian 2-cube is a homotopy push out. A basic notion in $[17,18]$ is the following definition.

(4.5) Definition. Let $D$ be a homotopy functor as in (4.1). Then $D$ is termed $n$ excisive if $D(\underline{X})$ is Cartesian for every strongly co-Cartesian $(n+1)$-cube $\underline{X}$ in space $_{r}$. We say that $D$ is linear if $D$ is reduced and 1-excisive and we say that $D$ is quadratic if $D$ is reduced and 2-excisive.

Goodwillie [17] proved the following result:

(4.6) Theorem. Let $D$ be a homotopy functor as in (4.1) which maps to space 1 or spectra and let $D$ be linear. Then D-homology is a homology theory.

We obtain the corresponding result for the quadratic case as follows.

(4.7) Theorem. Let $D$ be a homotopy functor as in (4.1) which maps to space $_{2}$ or spectra and let $D$ be quadratic. Then D-homology is a quadratic homology theory on pair $_{r}$ in the sense of (3.2).

Here we use space ${ }_{2}$ since we want all $D$-homology groups to be abelian.

Remark. Brown's representability theorem (see 2.8) shows that the conclusion in (4.6) admits a converse in the sense that each homology theory restricted to the category of finite $\mathrm{CW}$-complexes is the $D$-homology for an appropriate linear homotopy functor $D$. We do not know whether there is such a converse for theorem (4.7) as well. One might need additional axioms to characterize the quadratic homology theories obtained from quadratic homotopy functors.

Proof of (4.7). We obtain (3.2) (i) by (4.3) and (3.2) (ii) by (4.9) below; see also (4.11). Moreover (3.2) (iii) is proved in (4.12) below. 
We need the following lemma; compare 1.18 [18]. Let $\underline{X}, \underline{Y}$ be $n$-cubes in space and let $f: \underline{X} \rightarrow \underline{Y}$ be a map between $n$-cubes which may be considered to be a $(n+1)$-cube. Let hofib $(f)$ be the $n$-cube obtained by taking the homotopy fibers of $f(K): X(K) \rightarrow Y(K), K \in \mathbf{P}(n)$.

(4.8) Lemma. Assume $Y(\phi)$ is a connected space. Then $f$ is a Cartesian $(n+1)$ cube if and only if hofib $(f)$ is a Cartesian $n$-cube.

Below we shall also consider quadratic homotopy functors $D$ which map to space $_{1}$ and not necessarily to space ${ }_{2}$ as assumed in (4.7). For such homotopy functors the following two lemmas hold.

(4.9) Lemma. Let $D$ be a quadratic homotopy functor as in (4.1) which maps to space $_{1}$ or spectra. Then D-homology defines for $n \in \mathbb{Z}$ a quadratic functor

$$
H_{n}(-; D): \text { space }_{r} \rightarrow \mathbf{G r}
$$

which carries $X$ to $H_{n}(X, D)$. Moreover the suspension theory of cross effects $H_{n}(X \mid Y ; D)$ defined as in (2.11) is a homology theory.

The lemma is a consequence of Goodwillie's results. In fact, let $D(X \vee Y)_{2}$ be the homotopy fiber of $D\left(r_{2}\right): D(X \vee Y) \rightarrow D(Y)$ and let $D(X \mid Y)$ be the homotopy fiber of $D\left(r_{1}\right): D(X \vee Y)_{2} \rightarrow D(X)$. Then one readily checks that one has

$$
H_{n}(X \mid Y, D)=\pi_{n} D(X \mid Y)
$$

for any reduced homotopy functor $D$. If $D$ maps to space ${ }_{1}$ then $D(X \vee Y)_{2}$ is always a connected space. We now repeat an argument of Goodwillie which proves (4.9).

Proof of (4.9). We first show that the functor $D_{X}$ with $D_{X}(Y)=D(X \vee Y)_{2}$ is 1-excisive. In fact, let $\underline{Y}$ be a co-Cartesian 2-cube. Then $r_{2}: X \vee \underline{Y} \rightarrow \underline{Y}$ is a strongly co-Cartesian 3-cube; compare (A.1). Hence $D\left(r_{2}\right)=D(X \vee \underline{Y} \rightarrow \underline{Y})$ is a Cartesian 3-cube since $D$ is 2-excisive. Therefore (4.8) shows that $D_{X}(\underline{Y})$ is Cartesian and hence $D_{X}$ is 1-excisive. Now $D(X \mid Y)$ is the homotopy fiber of $D_{X}(Y) \rightarrow D_{X}(*)$. Therefore the functor $Y \longmapsto D(X \mid Y)$ is linear since $D_{X}$ is 1-excisive. This completes the proof of (4.9) by using (4.6).

(4.11) Remark. Let $D$ be a quadratic homotopy functor as in (4.1) which maps to space $_{1}$. Goodwillie showed that there exists a spectrum $E$ and a natural isomorphism

$$
H_{n}(X \mid Y ; D)=\pi_{n}(E \wedge X \wedge Y)
$$

which is compatible with the suspension $s$ of $X$ and the suspension $\bar{s}$ of $Y$; see (2.11), (2.12). Moreover the isomorphism is compatible with the interchange map on both sides. Here the interchange map $T$ on $\pi_{n}(E \wedge X \wedge Y)$ is introduced by a $\Sigma_{2}$-action $t$ on $E$ and by the interchange $T_{X, Y}: X \wedge Y \approx Y \wedge X$, that is $T=\pi_{n}\left(t \wedge T_{X, Y}\right)$. Let $(E \wedge X \wedge X)_{T}$ be the homotopy orbit spectrum given by $T$. Then the Goodwillie tower of the quadratic functor $D$ yields a natural fibration sequence

$$
\Omega^{\infty}(E \wedge X \wedge X)_{T} \rightarrow D(X) \rightarrow D^{\operatorname{lin}}(X) .
$$

Here the linearization satisfies $D^{\operatorname{lin}}(X)=\Omega^{\infty}\left(E^{\prime} \wedge X\right)$ for an appropriate spectrum $E^{\prime}$. 
(4.12) Lemma. Let $D$ be a quadratic homotopy functor as in (4.1) which maps to space $_{1}$ or spectra. Then for $Q_{n}(X, Y)=H_{n}(X, Y ; D)$ there exists a quadratic excision sequence as in (3.2) (iii) which is natural and exact.

We point out that in the lemma $Q_{1}(X, Y)=H_{1}(X, Y ; D)$ is only a pointed set if $D$ maps to space $_{1}$.

Proof of (4.12). Let $(X, A)$ be an object in pair $r_{r}$ and let $f: A \rightarrow X$ be the inclusion. Then $(X, A)$ yields a 3 -cube in space $_{r}$ termed $C u b e(X, A)$ which is obtained as the following map $F$ between 2-cubes

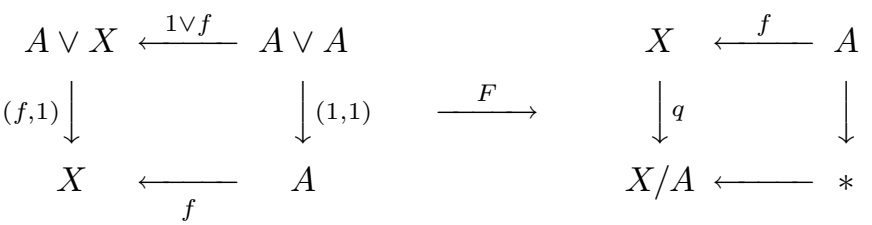

Here $F$ is defined by $(0,1): A \vee X \rightarrow X,(0,1): A \vee A \rightarrow A, q: X \rightarrow X / A$ and $0: A \rightarrow *$. One readily checks that $F=C u b e(X, A)$ is well defined. Each square in $C u b e(X, A)$ is a homotopy push out so that $C u b e(X, A)$ is actually strongly co-Cartesian. Since $D$ is quadratic this implies that $D(C u b e(X, A))$ is Cartesian and therefore by (4.8) the following diagram of homotopy fibers is a homotopy pull back.

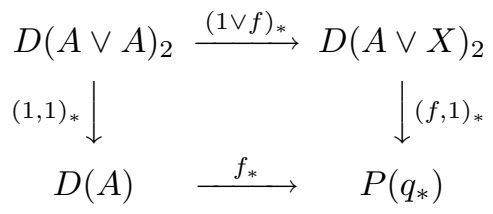

Here $P\left(q_{*}\right)$ is the homotopy fiber of $q_{*}: D(X) \rightarrow D(X / A)$. Let $K(A)$ be the homotopy fiber of $(1,1)_{*}: D(A \vee A)_{2} \rightarrow D(A)$. Then we get the fiber sequence

$$
K(A) \stackrel{(1 \vee f)_{*}}{\longrightarrow} D(A \vee X)_{2} \stackrel{(f, 1)_{*}}{\longrightarrow} P\left(q_{*}\right)
$$

since (2) is a homotopy pull back. This fiber sequence of spaces or spectra induces a long exact sequence of homotopy groups. The definition of $K(A)$ yields for $n \in \mathbb{Z}$ the following commutative diagram of short exact sequences of groups.

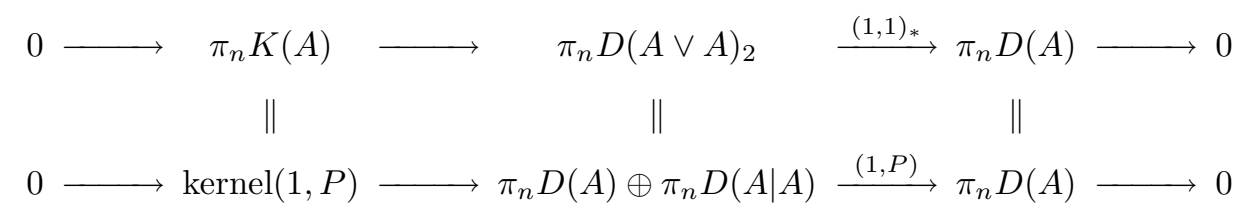

Here we use the isomorphism in (4.10) and (2.4) (4) and we point out that for $D:$ space $_{r} \rightarrow$ space $_{1}$ the fiber $D(A \vee X)_{2}$ is connected and that $\pi_{1}(D(A \mid A))$ is central and split in $\pi_{1} D(A \vee X)_{2}$. Now an isomorphism of abelian groups

$$
\Theta: \pi_{n} D(A \mid A) \cong \operatorname{kernel}(1, P)=\pi_{n} K(A)
$$

is obtained by mapping $y$ to $(P y,-y)$.

The boundary operator $\partial$ of the long exact sequence of homotopy groups associated to (3) yields the natural transformation

$$
\delta: H_{n+1}\left(C A \cup_{A} X, X ; D\right)=\pi_{n} P\left(q_{*}\right) \stackrel{\partial}{\longrightarrow} \pi_{n-1} K(A)=H_{n-1}(A \mid A ; D) .
$$

This completes the proof of the quadratic excision axiom. 
We can use (4.12) for the proof of the following result.

(4.13) Proposition. Let $D:$ space $_{1} \rightarrow$ space $_{1}$ be a quadratic homotopy functor. Then we obtain by (3.1) and (4.9) the square group

$$
H_{1}\left\{S^{1} ; D\right\}=\left(H_{1}\left(S^{1} ; D\right) \stackrel{H}{\longrightarrow} H_{1}\left(S^{1} \mid S^{1} ; D\right) \stackrel{P}{\longrightarrow} H_{1}\left(S^{1}, D\right)\right)
$$

and using the tensor product in (6.3) below there is a natural isomorphism

$$
H_{1}(Y, D) \cong \pi_{1}(Y) \otimes H_{1}\left\{S^{1} ; D\right\}
$$

for 2-dimensional $C W$-complexes $Y$.

Proof. We may assume that $Y=C A \cup_{A} X$ where $A$ and $X$ have the homotopy type of one point union of 1 -spheres. We now apply (4.12) and $\partial$-exactness. This gives us the exact sequence of groups, $Q_{1}(X)=H_{1}(X, D)$,

$$
Q_{1}(A \vee X)_{2} \rightarrow Q_{1}(X) \rightarrow Q_{1}(Y) \rightarrow 0 .
$$

Since $D$ preserves filtered colimits up to homotopy we get by [10] with $M=$ $H_{1}\left\{S^{1} ; D\right\}$ :

$$
\begin{aligned}
Q_{1}(X) & =\pi_{1}(X) \otimes M, \\
Q_{1}(A \vee X)_{2} & =Q_{1}(A) \oplus Q_{1}(A \mid X) \\
& =\pi_{1}(A) \otimes M \oplus \pi_{1}(A)^{a b} \otimes \pi_{1}(X)^{a b} \otimes M_{e e}
\end{aligned}
$$

and therefore the result follows from (8.4) [10]. For this observe that $\pi_{1}(A) \stackrel{d}{\rightarrow}$ $\pi_{1}(X) \stackrel{q}{\rightarrow} \pi_{1}(Y)$ has the property that $q$ is surjective and the normal closure of image of $d$ is the kernel of $q$.

(4.14) Example. It follows from Goodwillie's Calculus $[19,20]$ that there is a sequence of functors $P_{n}$ from pointed spaces to pointed spaces and natural transformations

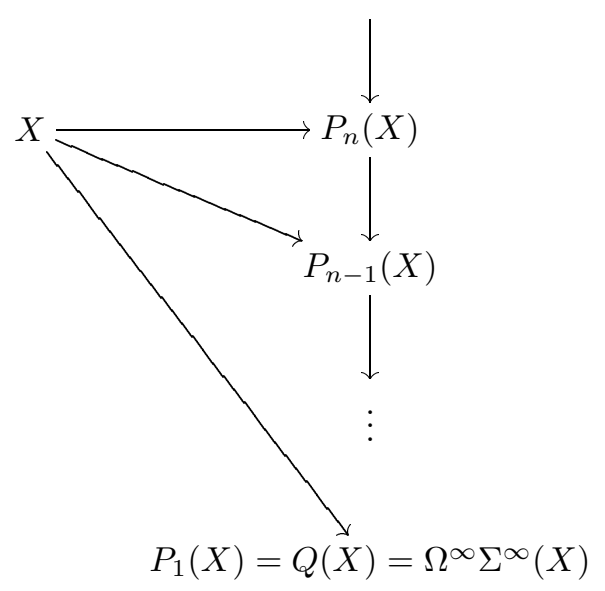

Here $P_{n}$ are homotopy functors satisfying $n$-th order excision and the maps $X \rightarrow$ $P_{n}(X)$ are $(n+1) k+1$ connected, where $k$ is the connectivity of $X$. The functors $P_{n}$ are uniquely determined by these universal properties. According to Goodwillie 
the tower above is termed the Taylor tower of the identity. Here $P_{1}$ is a linear homotopy functor and the homology

$$
H_{n}\left(X, P_{1}\right)=\pi_{n}^{S}(X)
$$

coincides with stable homotopy groups. Therefore the quadratic homotopy functor $P_{2}$ yields a canonical quadratic homology which we call quadratic homotopy groups:

$$
H_{n}\left(X, P_{2}\right)=\pi_{n}^{Q}(X) .
$$

This is the quadratic analogue of stable homotopy groups. We know by (4.7) that quadratic homotopy groups $\pi_{n}^{Q}$ form a quadratic homology theory on space ${ }_{2}$ satisfying all properties in section 3 . The cross effect of $\pi_{n}^{Q}(X)$ is given by a natural isomorphism

$$
\pi_{n}^{Q}(X \mid Y)=\pi_{n+1}^{S}(X \wedge Y)
$$

where $X \wedge Y=X \times Y / X \vee Y$, compare [19], [24]. Arone-Mahowald [1] mentioned that $P_{2}(X)$ also can be constructed by the fiber of the stable James-Hopf map $\gamma_{2}$ in [13]; that is

$$
P_{2}(X) \rightarrow Q(X) \stackrel{\gamma_{2}}{\longrightarrow} Q(X \wedge X)_{T}
$$

is a natural fiber sequence. Here $(X \wedge X)_{T}$ is the homotopy orbit space of the $\mathbb{Z} / 2$-action on $X \wedge X$ given by the interchange map $T_{X, X}$. In the metastable range we have considered examples of the quadratic $\mathbb{Z}$-modules $\pi_{n+k}^{Q}\left\{S^{n}\right\}$ in table 2 in 9.9 [5]. Such quadratic $\mathbb{Z}$-modules seem to be the appropriate quadratic analogue of stable homotopy groups of spheres.

\section{Homotopy FUnCTORS INDUCED BY ENDOFUNCTORS OF THE CATEGORY OF GROUPS}

Let $\mathbf{G r}$ be the category of groups and let $s \mathbf{G r}$ be the category of simplicial groups. A functor $F: \mathbf{G r} \rightarrow \mathbf{G r}$ induces the functor

$$
\bar{F}: s \mathbf{G r} \rightarrow s \mathbf{G r}
$$

which carries the simplicial group $X$ to the simplicial group $F \circ X$. We now consider the following composition of functors $F_{\sharp}=\beta \bar{F} \alpha$ :

$$
\text { space }_{1} \stackrel{\alpha}{\longrightarrow} s \mathbf{G r} \stackrel{\bar{F}}{\longrightarrow} s \mathbf{G r} \stackrel{\beta}{\longrightarrow} \text { space }_{1} .
$$

Here we obtain $\alpha$ as follows. For a pointed space $X$ let $S(X)$ be the reduced singular set consisting of all singular simplexes $\sigma: \Delta^{n} \rightarrow X$ with $\sigma(v)=*$ for all vertices $v$ of the simplex $\Delta^{n}$. Then the functor $\alpha$ carries $X$ to the Kan-loop group $\alpha(X)=G S(X)$ of $S(X)$; see for example [15]. Moreover for a simplical group $G$ let $|G|$ be the realization and let $\beta(G)=B|G|$ be the classifying space of the topological group $|G|$. Then $\alpha$ and $\beta$ induce equivalences of homotopy categories

$$
\text { space }_{1} / \simeq \underset{\beta}{\stackrel{\alpha}{\rightleftarrows}} H o(s \mathbf{G r})
$$

where $\beta$ is the inverse of $\alpha$. Here $H o(s \mathbf{G r})$ is the localization of $s \mathbf{G r}$ with respect to weak equivalences. Let $\mathbf{g r}$ be the full subcategory of $\mathbf{G r}$ consisting of free groups. Since $\alpha(X)$ above is actually a free simplicial group we see that $F_{\sharp}$ depends only on the restriction $F_{0}: \mathbf{g r} \rightarrow \mathbf{G r}$ of $F$. On the other hand each functor $F_{0}: \mathbf{g r} \rightarrow \mathbf{G r}$ determines a unique extension $F_{1}: \mathbf{G r} \rightarrow \mathbf{G r}$ with the property that $F_{1}$ preserves cokernels. Therefore we may assume that $F$ in (5.1) preserves cokernels. 
Moreover it is convenient to assume that the behaviour of $F_{\sharp}$ on infinite CWcomplexes is determined by its behaviour on finite complexes. Therefore we assume that $F_{\sharp}$ preserves filtered colimits up to homotopy. One readily checks that this is the case if and only if $F$ preserves filtered colimits. These remarks lead to the

(5.3) Definition. A group functor is an endofunctor $F: \mathbf{G r} \rightarrow \mathbf{G r}$ of the category of groups which preserves coequalizers and filtered colimits. Hence such a group functor is determined by its restriction to the full subcategory of finitely generated free groups.

(5.4) Lemma. A group functor $F: \mathbf{G r} \rightarrow \mathbf{G r}$ induces a functor $F_{\sharp}:$ space $_{1} \rightarrow$ space $_{1}$ which is a reduced homotopy functor.

We point out that the lemma does not imply that the functor $\bar{F}$ in (5.1) carries weak equivalences in $s \mathbf{G r}$ to weak equivalences in $s \mathbf{G r}$; this in general does not hold.

Proof of (5.4). Clearly $* \rightarrow F_{\sharp}(*)$ is a homotopy equivalence so that $F_{\sharp}$ is reduced. We have to show that $F_{\sharp}$ carries homotopy equivalences to weak equivalences. Let $H: f_{0} \simeq f_{1}$ be a homotopy in space ${ }_{1}$. Then there exists a homotopy $H^{\prime}$ : $\alpha f_{0} \simeq \alpha f_{1}$ since $\alpha(X)$ is a free simplicial group. The functor $\bar{F}$ carries $H^{\prime}$ to a homotopy $\bar{F} \alpha f_{0} \simeq \bar{F} \alpha f_{1}$ in the category of simplicial sets, see (1.10) and (4.2) in [15]. This implies that $\pi_{n}\left(\bar{F} \alpha f_{0}\right)=\pi_{n}\left(\bar{F} \alpha f_{1}\right)$ and hence $\pi_{n}\left(F_{\sharp} f_{0}\right)=\pi_{n}\left(F_{\sharp} f_{1}\right)$ for $n \in \mathbb{Z}$. From this one readily derives that $F_{\sharp}$ carries homotopy equivalences to weak equivalences.

(5.5) Definition. Let $F: \mathbf{G r} \rightarrow \mathbf{G r}$ be a group functor and let $X \in$ space $_{1}$. We define the F-homology of $X$,

$$
H_{n}(X ; F)=H_{n}\left(X ; F_{\sharp}\right)=\pi_{n}\left(F_{\sharp} X\right),
$$

by the $F_{\sharp}$-homology of $X, n \in \mathbb{Z}$. Similarly we obtain the relative $F$-homology $H_{n}(X, Y ; F)$ of a pair $(X, Y) \in$ pair $_{1}$. Clearly $F$-homology has the properties of $D$-homology described in (4.2) and (4.4). In particular $H_{1}(X ; F)$ is only a group; see (5.11). Moreover we obtain the stable F-homology

$$
H_{n}^{S}(X, F)=H_{n}\left(X, F_{\sharp}^{l i n}\right) .
$$

Compare (4.3). Let $F, G: \mathbf{G r} \rightarrow \mathbf{G r}$ be group functors. Then a natural transformation $t: F \rightarrow G$ induces natural transformations

$$
\begin{aligned}
& t_{\sharp}: F_{\sharp}(X) \rightarrow G_{\sharp}(X) \quad \text { and } \\
& t_{*}: H_{n}(X, Y ; F) \rightarrow H_{n}(X, Y ; G)
\end{aligned}
$$

where $t_{*}$ is the coefficient homomorphism induced by $t_{\sharp}$. We obtain $t_{\sharp}$ as follows. Let $\bar{t}: \bar{F} \rightarrow \bar{G}$ be the transformation induced by $F$. Then we set $t_{\sharp}=\beta \bar{t}_{\alpha X}$. Clearly the coefficient homomorphism $t_{*}$ is compatible with the boundary map $\partial$ of $F$-homology.

(5.6) Lemma. Let $(X, Y) \in$ pair $_{1}$ be an $r$-connected pair. Then $H_{n}(X, Y ; F)=0$ for $n \leq r$. This implies that

$$
H_{n}(X ; F)=H_{n}\left(X^{n+1} ; F\right)
$$

depends only on the $(n+1)$-skeleton of $X$. Moreover if $X$ is r-connected then so is $F_{\sharp}(X)$. 
Proof. We may assume that $X$ is a reduced CW-complex with subcomplex $Y$ and that $X-Y$ has only cells in dimension $>r$. Recall the notion of a "free simplicial group" as defined in section 4 of [15]. By a result of Kan [25] we obtain a homotopy equivalence

$$
\varphi:\left(G_{X}, G_{Y}\right) \simeq(\alpha X, \alpha Y)
$$

of pairs of free simplicial groups. Here $G_{X}$ as a free simplicial group has generators in degree $t$ which are exactly the $(t+1)$-cells of $X, t \geq 0$. Moreover $G_{Y}$ is the subobject generated by the cells of $Y$. Hence $\left(G_{X}\right)_{n}=\left(G_{Y}\right)_{n}$ for $n<r$ and therefore also $\left(\bar{F} G_{X}\right)_{n}=\left(\bar{F} G_{Y}\right)_{n}$ for $n<r$. Since $\bar{F}$ carries $\varphi$ above to a weak equivalence we see that $H_{n}(X, Y ; F)=0$ for $n \leq r$.

Let $I$ be the identity functor of the category $\mathbf{G r}$. Then one has the canonical natural isomorphism

$$
\pi_{n}(X, Y)=H_{n}(X, Y ; I)
$$

which we use as an identification. For a group $G$ let $\Gamma_{k}(G) \subset G$ be the subgroup of $k$-fold commutators. Then $G / \Gamma_{2} G=\operatorname{ab}(G)=G^{\text {ab }}$ is the abelianization of $G$. For $k \geq 1$ we obtain the nilization functors

$$
n i l_{k}: \mathbf{G r} \rightarrow \mathbf{G r}
$$

which carry $G$ to the quotient $n i l_{k}(G)=G / \Gamma_{k+1} G$. Hence $n i l_{1}=a b$ is the abelianization functor. All functors $n i l_{k}, k \geq 1$, are group functors in the sense of (5.3). By the result of Dold-Kan it is well known that for $(X, Y) \in$ pair $_{1}$ one has the natural isomorphism

$$
H_{n}(X, Y ; a b)=H_{n}(X, Y)
$$

where the right hand side is the singular homology. Moreover the natural transformation $I \rightarrow a b$ given by the quotient map $G \rightarrow a b(G)$ induces the classical Hurewicz homomoprhism

$$
\pi_{n}(X, Y)=H_{n}(X, Y ; I) \rightarrow H_{n}(X, Y ; a b)=H_{n}(X, Y) .
$$

Similarly one obtains by the natural quotient map $I \rightarrow n i l_{k}(G)$, the $n i l_{k}$-Hurewicz homomorphism

$$
\pi_{n}(X, Y)=H_{n}(X, Y ; I) \rightarrow H_{n}\left(X, Y ; n i l_{k}\right) .
$$

The following generalization of the classical Hurewicz theorem is due to Curtis [15].

(5.10) Curtis theorem. Let $r \geq 2$ and let $X$ be an $(r-1)$-connected space. Then the nil $_{k}$-Hurewicz homomorphism

$$
\pi_{n}(X) \rightarrow H_{n}\left(X ; n i l_{k}\right)
$$

is an isomorphism for $n<r+\left\{\log _{2}(k+1)\right\}$ and is surjective for $n=r+\left\{\log _{2}(k+1)\right\}$. Here $\{a\}$ denotes the least integer $\geq a$.

The Hurewicz theorem is the case $k=1$ of this result since $\left\{\log _{2}(2)\right\}=\{1\}=1$. For $k=2$ we have $\left\{\log _{2}(3)\right\}=2$ since $2>\log _{2}(3)>1$. Hence the $n i l_{2}$-Hurewicz homomorphism yields for an $(r-1)$-connected space $X, r \geq 2$,

$$
\begin{aligned}
\pi_{r}(X) & =H_{r}\left(X, n i l_{2}\right), \\
\pi_{r+1}(X) & =H_{r+1}\left(X, n i l_{2}\right), \\
\pi_{r+2}(X) & \rightarrow H_{r+2}\left(X, n i l_{2}\right),
\end{aligned}
$$


where $\rightarrow$ denotes a surjection. Clearly the functor $n i l_{1}=a b$ is linear and the functor $n i l_{2}$ is quadratic in the sense of (2.9). In the next section we consider all quadratic group functors $\mathbf{G r} \rightarrow \mathbf{G r}$. It would be interesting to understand the stable theory $H_{n}^{S}\left(X, n i l_{k}\right), k \geq 1$, which is a homology theory approximating for $k \rightarrow \infty$ stable homotopy $\pi_{n}^{S}(X)$. We shall determine $H_{n}^{S}(X, F)$ for any quadratic group functor in (8.5). This in particular yields an explicit spectrum $E$ for which $H_{n}^{S}\left(X, n i l_{2}\right)=H_{n}(X, E)$; compare (8.16).

The next result corresponds to (4.13) in case $F$ is a quadratic group functor.

(5.12) Lemma. Let $F: \mathbf{G r} \rightarrow \mathbf{G r}$ be a group functor. Then we have for $X \in$ space $_{1}$ the natural isomorphism

$$
H_{1}(X, F)=F\left(\pi_{1}(X)\right) .
$$

Moreover if $S$ is a one point union of 1-spheres we have

$$
H_{n}(S, F)=0 \quad \text { for } \quad n \geq 2 .
$$

Proof. The group $G=\pi_{1}(S)$ is a free group for which the constant simplicial group $\bar{G}$ with $\bar{G}_{n}=G$ for $n \geq 0$ is a free simplicial group which is homotopy equivalent to $\alpha(S)$. Hence one has a weak equivalence $\bar{F}(\bar{G}) \rightarrow \bar{F} \alpha(S)$ where $\bar{F}(\bar{G})$ is again a constant simplicial group. This shows $H_{n}(S, F)=\pi_{n-1} \bar{F}(\bar{G})=0$ for $n \geq 2$ and $H_{1}(S, F)=\pi_{1} \bar{F}(\bar{G})=F(G)$. Now let $H=\alpha X$ with $\pi_{0} H=\pi_{1} X$. Then the degree 1 part of the simplicial group $H$

$$
H_{1} \rightrightarrows H_{0} \rightarrow \pi_{0} H
$$

is a coequalizer. Since $F$ preserves cokernels also

$$
F H_{1} \rightrightarrows F H_{0} \rightarrow F \pi_{0} H
$$

is a coequalizer and therefore $\pi_{0} F H=F \pi_{0} H$. This shows $H_{1}(X, F)=F\left(\pi_{1} X\right)$.

If $F=a b$ is the abelianization functor we have by (5.9) and (5.11)

$$
H_{1}(X)=H_{1}(X, a b)=a b\left(\pi_{1}(X)\right) .
$$

This is a part of the classical Hurewicz theorem.

\section{Square-homology}

We show that the homology theories obtained by linear group functors $\mathbf{G r} \rightarrow \mathbf{G r}$ are exactly the classical "ordinary homology theories" of Eilenberg-Mac Lane. This motivates the study of homology defined by quadratic group functors $\mathbf{G r} \rightarrow \mathbf{G r}$. There is the following classification of linear functors in [10].

(6.1) Lemma. The category of linear group functors $\mathbf{G r} \rightarrow \mathbf{G r}$ is equivalent to the category $\mathbf{A b}$ of abelian groups. More precisely for each linear group functor $F$ there is an abelian group $A$ and an isomorphism

$$
F(G)=a b(G) \otimes A
$$

which is natural in $G \in \mathbf{G r}$. The equivalence carries $F$ to $A=F(\mathbb{Z})$. In particular $F$ admits a factorization

$$
F: \mathbf{G r} \stackrel{a b}{\longrightarrow} \mathbf{A b} \stackrel{\otimes A}{\longrightarrow} \mathbf{A} \mathbf{b} \subset \mathbf{G r} .
$$

As a consequence of this result and the Dold-Kan theorem [16] we get 
(6.2) Proposition. Let $F$ be a linear group functor corresponding to $A \in \mathbf{A b}$ as in (6.1). Then one has for $(X, Y) \in$ pair $_{1}$ the natural isomorphism, $n \in \mathbb{Z}$,

$$
H_{n}(X, Y ; F)=H_{n}(X, Y ; A)
$$

where the right hand side is the singular homology with coefficients in $A$.

This implies that the homology theories of linear group functors are exactly the ordinary homology theories in the sense of Eilenberg-Steenrod which satisfy the classical "dimension axiom". As a next step we consider quadratic group functors. For this recall from [10] the following notation on square groups.

(6.3) Definition. A square group

$$
M=\left(M_{e} \stackrel{H}{\longrightarrow} M_{e e} \stackrel{P}{\longrightarrow} M_{e}\right)
$$

is given by a group $M_{e}$ and an abelian group $M_{e e}$. Both groups are written additively. Moreover $P$ is a homomorphism and $H$ is a quadratic function, that is the cross effect

$$
(a \mid b)_{H}=H(a+b)-H(b)-H(a)
$$

is linear in $a, b \in M_{e}$. In addition the following properties are satisfied $\left(x, y \in M_{e e}\right)$.

$$
\begin{aligned}
& (P x \mid b)_{H}=0 \quad \text { and } \quad(a \mid P y)_{H}=0, \\
& P(a \mid b)_{H}=a+b-a-b, \\
& P H P(x)=P(x)+P(x),
\end{aligned}
$$

By (1) and (2) $P$ maps to the center of $M_{e}$ and by (2) the cokernel of $P$ is abelian. Hence $M_{e}$ is a group of nilpotency degree 2 . Let Square be the category of square groups. As an example we have the square group

$$
\mathbb{Z}_{\text {nil }}=(\mathbb{Z} \stackrel{H}{\longrightarrow} \mathbb{Z} \stackrel{0}{\longrightarrow} \mathbb{Z})
$$

with $H(r)=\left(\begin{array}{l}r \\ 2\end{array}\right)$ and $P=0$; many other examples are discussed in $[9,10]$. A quadratic $\mathbb{Z}$-module $M$ is a square group for which $H$ is linear and $H P H=2 H$; see (3.1).

Let $G$ be a group and let $M$ be a square group. Similarly as in (3.10) we define the group $G \otimes M$ by the generators $g \otimes a$ and $[g, h] \otimes x$ with $g, h \in G, a \in M_{e}$ and $x \in M_{e e}$ subject to the relations

$$
\begin{aligned}
(g+h) \otimes a & =g \otimes a+h \otimes a+[g, h] \otimes H(a), \\
{[g, g] \otimes x } & =g \otimes P(x)
\end{aligned}
$$

where $g \otimes a$ is linear in $a$ and where $[g, h] \otimes x$ is central and linear in each variable $g, h$ and $x$. There are obvious induced maps for this tensor product so that one gets a bifunctor

$$
\otimes: \mathbf{G r} \times \text { Square } \rightarrow \text { Gr. }
$$

One has the natural isomorphism

$$
n i l_{2}(G)=G \otimes \mathbb{Z}_{n i l} .
$$

In $[5,10]$ we obtain the following classification of quadratic group functors which is the quadratic analog of (6.1). Let Nil be the category of groups of nilpotency degree 2. 
(6.4) Proposition. The category of quadratic group functors $\mathbf{G r} \rightarrow \mathbf{G r}$ is equivalent to the category Square of square groups. More precisely for each quadratic group functor $F$ one has the square group $M=F\{\mathbb{Z}\}$ in (3.1) and isomorphisms

$$
F(G)=G \otimes M=n i l_{2}(G) \otimes M
$$

which are natural in $G \in \mathbf{G r}$. The equivalence carries $F$ to $M$ and $F=$ nil $_{2}$ corresponds to $M=\mathbb{Z}_{n i l}$. In particular quadratic group functors admit a factorization

$$
\mathrm{Gr} \stackrel{n i l_{2}}{\longrightarrow} \mathrm{Nil} \stackrel{\otimes M}{\longrightarrow} \mathrm{Nil} \subset \mathbf{G r} .
$$

Moreover the quadratic group functors which admit a factorization

$$
\mathbf{G r} \stackrel{a b}{\longrightarrow} \mathbf{A b} \longrightarrow \mathbf{A b} \subset \mathbf{G r}
$$

correspond exactly to quadratic $\mathbb{Z}$-modules $M$.

Using this result we identify square groups $M$ and quadratic group functors $\mathbf{G r} \rightarrow \mathbf{G r}$; we write $M: \mathbf{G r} \rightarrow \mathbf{G r}$ for the functor which carries $G$ to $G \otimes M$. As a quadratic analog of (6.2) we define for a square group $M$ and $(X, Y) \in$ pair $_{1}$ the square-homology with coefficients in $M, n \in \mathbb{Z}$,

$$
H_{n}(X, Y ; M)=H_{n}\left(X, Y ; M_{\sharp}\right)=\pi_{n}\left(M_{\sharp} X, M_{\sharp} Y\right) .
$$

Here $M_{\sharp}$ is the homotopy functor (5.1) induced by $M$. The groups $H_{n}(X ; M)$ and $H_{n+1}(X, Y ; M)$ are abelian for $n \geq 2$ and of nilpotency degree 2 for $n=1$. Moreover $H_{1}(X, Y ; M)$ is a pointed set. For $n \leq 0$ all groups (6.5) are trivial.

(6.6) Theorem. Let $F: \mathbf{G r} \rightarrow \mathbf{G r}$ be a quadratic group functor. Then $F_{\sharp}$ in (5.1) is a quadratic homotopy functor.

We prove this result in the Appendix A. Since for a simply connected space $X$ $F_{\sharp} X$ is also simply connected and we obtain by $(6.6),(6.4),(4.7)$ the corollary:

(6.7) Corollary. Square-homology $H_{n}(X, Y ; M)$ with coefficients in a square group $M$ is a quadratic homology theory on pair $_{2}$ in the sense of (3.2).

Using (4.3) we derive from (6.6) the next corollary which yields the homology theory or spectrum associated to a square group.

(6.8) Corollary. Stable square-homology $H_{n}^{S}(X, Y ; M)$ with coefficients in a square group $M$ is a homology theory on pair ${ }_{1}$.

In Corollary (6.7) we use the category space ${ }_{2}$ in order to obtain abelian groups $H_{n}(X, Y ; M)$. The next result improves (6.7) for the category space ${ }_{1}$.

(6.9) Theorem. Let $M$ be a square group. Then square-homology with coefficients in $M$ defines a quadratic functor

$$
H_{n}(-; M): \text { space }_{1} \rightarrow\left\{\begin{array}{lll}
\text { Ab } & \text { for } & n \geq 2, \\
\text { Nil } & \text { for } & n=1
\end{array}\right.
$$

with cross effect

$$
H_{n}(X \mid Y ; M)=H_{n+1}\left(X \wedge Y ; M_{e e}\right)
$$

for $n \in \mathbb{Z}$. Here the right hand side is the singular homology of the smash product $X \wedge Y$ with coefficients in the abelian group $M_{e e}$. The isomorphism is compatible 
up to sign with the suspension $s$ and $\bar{s}$ and the interchange map on both sides. Moreover for $(X, A) \in$ pair $_{1}$ one has the natural exact sequence of groups:

$$
\begin{gathered}
H_{n-1}(A ; M) \oplus H_{n}\left(A \wedge X ; M_{e e}\right) \\
\| \\
\stackrel{\delta}{\longrightarrow} H_{n}\left(A \wedge A ; M_{e e}\right) \stackrel{j_{\sharp}}{\longrightarrow} H_{n}(C A \vee X, A \vee X ; M) \\
\stackrel{i_{\sharp}}{\longrightarrow} H_{n}\left(C A \cup_{A} X, X ; M\right) \stackrel{\delta}{\longrightarrow} H_{n-1}\left(A \wedge A ; M_{e e}\right)
\end{gathered}
$$

The operators $i_{\sharp}$ and $j_{\sharp}$ are defined as in (3.2) (iii). For $n \leq 1$ the sequence consists of trivial groups. As in (3.5) we derive from the quadratic excision sequence in (6.9) the following long exact sequence.

(6.10) Corollary. Let $M$ be a square group and $A \in$ space $_{1}$. Then one has the natural long exact sequence of groups, $n \in \mathbb{Z}$,

$$
\stackrel{P}{\longrightarrow} H_{n}(A ; M) \stackrel{s}{\longrightarrow} H_{n+1}(\Sigma A ; M) \stackrel{H^{\prime}}{\longrightarrow} H_{n}\left(A \wedge A ; M_{e e}\right) \stackrel{P}{\longrightarrow} H_{n-1}(A ; M) \rightarrow .
$$

Proof of (6.9). In the following proof we do not use (6.6). Let $F: \mathbf{G r} \rightarrow \mathbf{G r}$ be given by $F(G)=G \otimes M$ where $M$ is the square group. By [10] we have for $X, Y \in \mathbf{G r}$ the binatural isomorphism

$$
F(X \mid Y)=a b(X) \otimes a b(Y) \otimes M_{e e} .
$$

We now consider the following commutative cubical diagram in $\mathbf{G r}$ which is determined by the inclusion

$$
f: A \rightarrow A \vee C=X
$$

where $X$ is the sum of $A$ and $C$ in $\mathbf{G r}$. The pair $(X, A)$ yields the three cube $G=C u b e(X, A)$ as in (4.12):

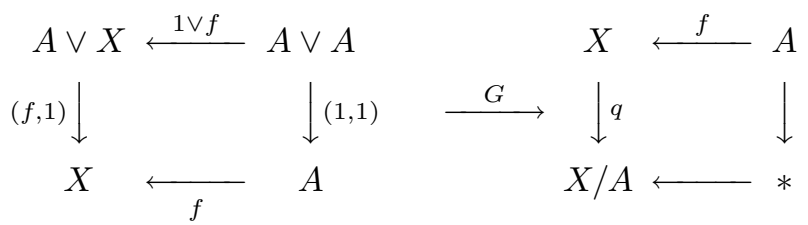

Here the map $G$ between 2-cubes is given by $(0,1): A \vee X \rightarrow X,(0,1): A \vee A \rightarrow A$, $q=(0,1): A \vee C=X \rightarrow X / A=C$ and $0: A \rightarrow *$. One readily checks that Cube $(X, A)$ is well defined. We now apply $F$ to $C u b e(X, A)$ and we obtain the following square consisting of the kernels of all $F(g)$ where $g$ is one of the arrows in $G$.

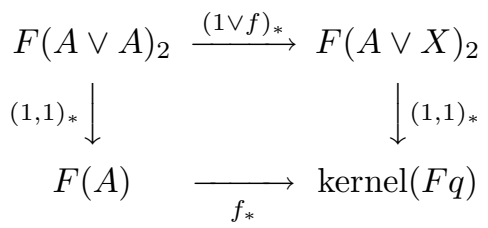

Using the assumption that $X=A \vee C$ we see by (1) that $(1 \vee f)_{*}$ in (4) induces an isomorphism of the kernels of the vertical arrows in (4). Hence (4) is a pull back diagram in Gr. Now let

$$
f: A \rightarrow X
$$


be a cofibration of simplicial groups. Then we know for all $n$ that we can choose free groups $C_{n}$ such that

$$
X_{n}=A_{n} \vee C_{n}
$$

Hence $f$ in (4) is of the form (2) for each $n$. This implies by the naturality of (3) and (4) that also (4) with $f$ as in (5) is a pull back diagram in the category of simplicial groups. This yields the short exact sequence of simplicial groups

$$
\begin{aligned}
& 0 \rightarrow K(A) \stackrel{(1 \vee f)^{*}}{\longrightarrow} F(A \vee X)_{2} \stackrel{(f, 1)_{*}}{\longrightarrow} \operatorname{kernel}(F q) \rightarrow 0, \\
& K(A)=\operatorname{kernel}\left\{(1,1)_{*}: F(A \vee A)_{2} \rightarrow F(A)\right\} .
\end{aligned}
$$

Using $F_{\sharp}$ in (5.1) we see that $H_{n}\left(X^{\prime} ; M\right)=\pi_{n-1}(F X)$ where $X=\alpha X^{\prime}$ is the simplicial group associated to $X^{\prime} \in$ space $_{r}$. Hence we get by (1) the cross effect formula

$$
\begin{aligned}
H_{n}\left(X^{\prime} \mid Y^{\prime} ; M\right) & =\pi_{n-1}(F(X \mid Y)) \\
& =\pi_{n-1}\left(a b(X) \otimes a b(Y) \otimes M_{e e}\right) \\
& =H_{n+1}\left(X^{\prime} \wedge Y^{\prime} ; M_{e e}\right) .
\end{aligned}
$$

Here the last isomorphism is given by the Eilenberg-Zilber theorem. This shows that the cross effect formula in (6.9) is satisfied. It only remains to check the quadratic excision sequence. Now a pair $\left(X^{\prime}, A^{\prime}\right)$ in pair ${ }_{1}$, corresponds to a cofibration $A \longmapsto$ $X$ in the category of simplicial groups for which we have the short exact sequence (7). This sequence induces a long exact sequence of homotopy groups which is isomorphic to the quadratic excision sequence for $\left(X^{\prime}, A^{\prime}\right)$. The boundary $\partial$ for $(7)$ determines the natural operator

$$
\begin{aligned}
\delta: H_{n+1}\left(C A^{\prime} \cup X^{\prime} ; M\right) & =\pi_{n-1} \operatorname{kernel}(F q) \stackrel{\partial}{\longrightarrow} \pi_{n-2} K(A) \\
& \stackrel{\Theta}{=} \pi_{n-2} F(A \mid A)=H_{n-1}\left(A^{\prime} \mid A^{\prime} ; M\right) .
\end{aligned}
$$

Here the definition of $K(A)$ in (8) yields the split short exact sequence

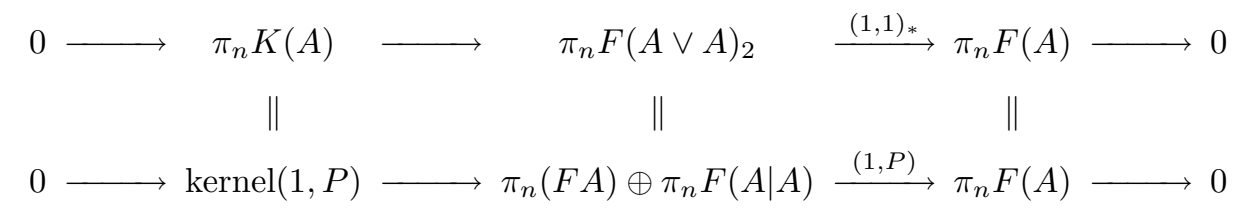

where we use the isomorphism in (2.9) (4). Now the isomorphism $\Theta$ in (10)

$$
\Theta: \pi_{n}(F(A \mid A)) \cong \operatorname{kernel}(1, P)=\pi_{n} K(A)
$$

is obtained by mapping $y$ to $(P y,-y)$. This corresponds to the definition of $j_{\sharp}$ in (3.2) (iii).

The first nonvanishing homology with coefficients in a square group is described by the following result.

(6.11) Proposition. Let $M$ be a square group and let $M^{a d d}=\operatorname{cok}(P) \in \underline{\underline{A b}}$ be the cokernel of $P: M_{e e} \rightarrow M_{e}$. If $X$ is an $(r-1)$-connected space one has

$$
H_{r}(X, M)=\left\{\begin{array}{l}
\pi_{1}(X) \otimes M \text { for } r=1, \\
\pi_{r}(X) \otimes M^{\text {add }} \text { for } r>1 .
\end{array}\right.
$$


Proof. For $r=1$ this is a consequence of (5.11) or (4.13). Now let $r \geq 2$. The EHP-sequence shows that for a one point union $S$ of 1 -spheres the sequence

$$
a b(G) \otimes a b(G) \otimes M_{e e} \stackrel{P}{\longrightarrow} G \otimes M \rightarrow H_{2}(\Sigma S ; M) \rightarrow 0
$$

is exact where $G=\pi_{1}(S)$. This implies

$$
H_{2}(\Sigma S ; M)=a b(G) \otimes M^{a d d} .
$$

Moreover the EHP-sequence shows that $H_{2}(\Sigma S ; M)=H_{n}\left(\Sigma^{n-1} S ; M\right)$ for $n \geq 2$. Now we have

$$
H_{r}(X ; M)=H_{r}\left(X^{r+1} ; M\right) .
$$

Here $X^{r+1}$ is the mapping cone of a maps $f: S^{\prime} \rightarrow S^{\prime \prime}$ where $S^{\prime}$ and $S^{\prime \prime}$ are one point unions of $r$-spheres. Now the $\partial$-exactness and the quadratic excision shows $H_{r}\left(X^{r+1} ; M\right)=\pi_{r}(X) \otimes M^{\text {add }}$.

\section{Square homology With Coefficients}

Let $s \mathbf{A b}$ be the category of simplicial abelian groups and let Chain be the category of chain complexes $C$ of abelian groups with $C_{i}=0$ for $i<0$. It is a result of Dold and Kan that there are isomorphisms of categories (see [9])

$$
s \mathbf{A b} \underset{K}{\stackrel{N}{\rightleftarrows}} \text { Chain }
$$

where $K$ is the inverse of the normalization $N$. Here $N(A)$ is also termed the Moore chain complex of $A$. Kan [26], 15.1, showed that for a reduced simplicial set $X$ and its Kan loop group $G(X)$ one has the isomorphism of chain complexes

$$
N(A(X)) \cong s^{-1} \tilde{C}_{*} X
$$

Here $A(X)=a b G(X)$ is the abelianization of $G(X)$ and $\tilde{C}_{*} X$ is the reduced (normalized) chain complex of the simplicial set $X$. Recall that the suspension $s^{k} C$ with $k \in \mathbb{Z}$ is $\left(s^{k} C\right)_{n}=C_{n-k}$ with the differential $d\left(s^{k} x\right)=(-1)^{k} s^{k}(d x)$. We define for $X \in$ space $_{1}$ the singular chain complex

$$
\tilde{C}_{*} X=\tilde{C}_{*}(S X)
$$

where $S X$ is the reduced singular set in (5.1). Given a quadratic $\mathbb{Z}$-module $M$ we obtain the induced chain functor $M_{\sharp}$ which is the composite

$$
M_{\sharp}: \text { Chain } \stackrel{K}{\longrightarrow} s \mathbf{A b} \stackrel{\otimes M}{\longrightarrow} s \mathbf{A b} \stackrel{N}{\longrightarrow} \text { Chain. }
$$

The next result is a consequence of (7.1), (7.2) and (6.4).

(7.4) Proposition. Let $M$ be a quadratic $\mathbb{Z}$-module and $X \in$ space $_{1}$. Then the square homology $H_{*}(X, M)$ is determined by $\tilde{C}_{*} X$. More precisely there is a natural isomorphism, $n \in \mathbb{Z}$,

$$
H_{n}(X, M)=H_{n-1}\left(M_{\sharp}\left(s^{-1} \tilde{C}_{*} X\right)\right) .
$$


Proof. Using the definitions we get

$$
\begin{aligned}
H_{n}(X, M) & =\pi_{n}\left(M_{\sharp} X\right) \\
& =\pi_{n-1}((G S X) \otimes M) \\
& =\pi_{n-1}\left((G S X)^{a b} \otimes M\right) \\
& =\pi_{n-1}\left(\left(K N(G S X)^{a b}\right) \otimes M\right) \\
& =\pi_{n-1}\left(\left(K s^{-1} \tilde{C}_{*} X\right) \otimes M\right) \\
& =H_{n-1} N\left(\left(K s^{-1} \tilde{C}_{*} X\right) \otimes M\right) \\
& =H_{n-1} M_{\sharp}\left(s^{-1} \tilde{C}_{*} X\right) .
\end{aligned}
$$

In (*) we use the fact that for a group $G$ we have $G \otimes M=G^{a b} \otimes M$ if $M$ is a quadratic $\mathbb{Z}$-module; compare (6.4).

(7.5) Remark. The homotopy type of the chain complex $\tilde{C}_{*} X$ is determined by the homology $H_{*}(X, \mathbb{Z})$. This shows by $(7.4)$ that the abelian groups $H_{n}(X, M)$ are determined by $H_{*}(X, \mathbb{Z})$ and $M$ provided $M$ is a quadratic $\mathbb{Z}$-module. This is not true if $M$ is a square group. For example we obtain by (5.11)

$$
0=H_{3}\left(\mathbb{C} P_{2}, \mathbb{Z}_{n i l}\right) \neq H_{3}\left(S^{2} \vee S^{4}, \mathbb{Z}_{n i l}\right)=\mathbb{Z}
$$

with $H_{*}\left(\mathbb{C} P_{2}, \mathbb{Z}\right)=H_{*}\left(S^{2} \vee S^{4}, \mathbb{Z}\right)$. Here $\mathbb{C} P_{2}$ is the complex projective plane and $S^{2} \vee S^{4}$ is the one point union of spheres.

(7.6) Remark. The universal coefficient formula in [11] can be used to compute $H_{*}(X, M)$ if $M$ is a quadratic $\mathbb{Z}$-module. For example if $M_{e e}$ is torsion free one has the natural short exact sequence

$$
0 \rightarrow\left(\bar{H}_{*} \otimes M\right)_{n-1} \rightarrow H_{n}(X, M) \rightarrow\left(\bar{H}_{*} *^{\prime} M\right)_{n-2} \rightarrow 0
$$

where $\bar{H}_{*}=s^{-1} \tilde{H}_{*}(X, \mathbb{Z})$ is the desuspended reduced homology of $X$. Here we use the graded tensor and torsion products defined in [11].

Now let $M$ be a square group. Then there is the canonical short exact sequence

$$
0 \rightarrow \hat{M} \stackrel{i}{\longrightarrow} M \stackrel{q}{\longrightarrow} M^{a d d} \rightarrow 0
$$

in the category Square where $M^{a d d}=\operatorname{cok}(P)$ is an abelian group and where $\hat{M}$ is a quadratic $\mathbb{Z}$-module. More precisely we obtain the commutative diagram

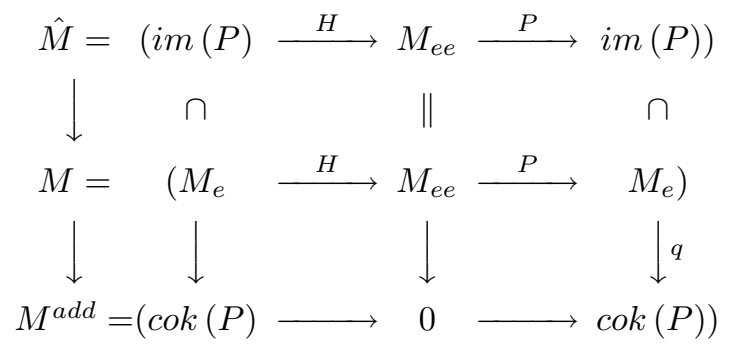

where $q$ is the quotient map. The definition of a square group shows readily that the restriction of $H$ in $M$ to $\hat{M}_{e}=i m(P)$ is a homomorphism and that $\hat{M}$ is a 
quadratic $\mathbb{Z}$-module. If $G$ is a free group then (7.7) induces the short exact sequence of groups

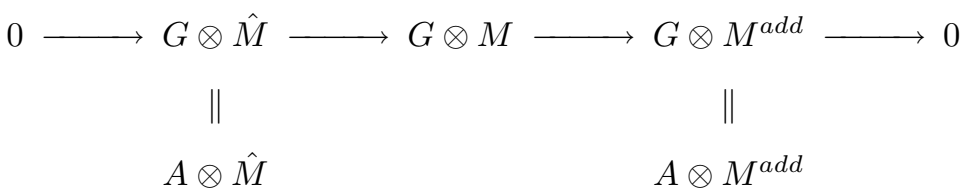

where $A=a b(G)$. If $G$ is a free simplicial group this sequence is still well defined and short exact. Hence the long exact sequence of homotopy groups for $G=G(S X)$ yields the following long exact sequence of square homology groups

$$
\stackrel{\partial}{\longrightarrow} H_{n}(X, \hat{M}) \stackrel{i_{*}}{\longrightarrow} H_{n}(X, M) \stackrel{q_{*}}{\longrightarrow} H_{n}\left(X, M^{\text {add }}\right) \stackrel{\partial}{\longrightarrow} H_{n-1}(X, \hat{M}) \longrightarrow .
$$

Here $H_{*}\left(X, M^{a d d}\right)$ is the usual homology of $X$ with coefficients in the abelian group $M^{a d d}=\operatorname{cok}\left(P: M_{e e} \rightarrow M_{e}\right)$. Moreover $H_{*}(X, \hat{M})$ depends by (7.5) only on the homology $H_{*}(X, \mathbb{Z})$ and $\hat{M}$. In fact $H_{*}(X, \hat{M})$ can be computed by the universal coefficient theorem [11] as follows; see (7.6).

Given a square group $M$ we obtain the involution

$$
T=H P-1: M_{e e} \rightarrow M_{e e}
$$

with $T T=1$. This yields the chain complex of groups

$$
M_{*}^{e}=\left(M_{e} \overleftarrow{P} M_{e e} \overleftarrow{1-T} M_{e e} \overleftarrow{1+T} M_{e e} \overleftarrow{1-T} \ldots\right)
$$

where $M_{e}$ is in degree 0 . We have $1-T=2-H P$ and $1+T=H P$ so that the homology of $M_{*}^{e}$ is

$$
H_{n}\left(M_{*}^{e}\right)= \begin{cases}\operatorname{cok}(P), & n=0, \\ \operatorname{ker}(P) / \operatorname{im}(2-H P), & n=1, \\ k \operatorname{ker}(2-H P) / \operatorname{im}(H P), & n=2 k \geq 2, \\ \operatorname{ker}(H P) / \operatorname{im}(2-H P), & n=2 k+1 \geq 3 .\end{cases}
$$

Moreover we associate with $M$ the following quadratic $\mathbb{Z}$-modules $Z_{n} M_{*}, n \geq 1$.

$$
Z_{n} M_{*}= \begin{cases}\operatorname{ker}(P) \stackrel{j}{\longrightarrow} M_{e e} \stackrel{2-H P}{\longrightarrow} \operatorname{ker}(P), & n=1, \\ \operatorname{ker}(2-H P) \stackrel{j}{\longrightarrow} M_{e e} \stackrel{H P}{\longrightarrow} \operatorname{ker}(2-H P), & n=2 k \geq 2, \\ \operatorname{ker}(H P) \stackrel{j}{\longrightarrow} M_{e e} \stackrel{2-H P}{\longrightarrow} \operatorname{ker}(H P), & n=2 k+1 \geq 3 .\end{cases}
$$

Here $j$ denotes the inclusion. We now compute the square-homology of a Moore space $M(A, n)$ of an abelian group $A$.

(7.10) Proposition. Let $n \geq 2$ and let $M$ be a square group. Then the square homology of the Moore space $M(A, n), A \in \mathbf{A b}$, has the following properties. If $A$ is free abelian there is a natural isomorphism

$$
H_{n+k}(M(A, n) ; M)=\left\{\begin{array}{l}
0 \text { for } k<0 \quad \text { or } k \geq n \\
A \otimes H_{k} M_{*}^{e} \text { for } 0 \leq k<n-1 \\
A \otimes Z_{n-1} M_{*} \text { for } k=n-1
\end{array}\right.
$$


In the general case one gets for $A \in \mathbf{A b}$ the following natural isomorphisms and short exact sequences respectively.

$$
\begin{aligned}
& H_{n+k}(M(A, n) ; M)= \begin{cases}0 & \text { for } k<0, k>n+1, \\
A \otimes \operatorname{cok} P, & \text { for } k=0, \\
A *^{\prime} Z_{n-1} M_{*} & \text { for } k=n, \\
A *^{\prime \prime} Z_{n-1} M_{*} & \text { for } k=n+1,\end{cases} \\
& 0 \rightarrow A \otimes Z_{n-1} M_{*} \rightarrow H_{2 n-1}(M(A, n) ; M) \rightarrow A * H_{n-2} M_{*}^{e} \rightarrow 0, \\
& 0 \rightarrow A \otimes H_{r} M_{*}^{e} \rightarrow H_{n+r}(M(A, n) ; M) \rightarrow A * H_{r-1} M_{*}^{e} \rightarrow 0
\end{aligned}
$$

for $0<r<n-1$.

In (8.4) we shall see that the short exact sequence for $H_{n+r}(M(A, n) ; M), 0<$ $r<n-1$, is actually naturally split.

(7.11) Remark. We now determine the spherical groups of square homology; see (3.8). Let $M$ be a square group. For each sphere $S^{n}$ we obtain by (3.1) the square group $(n \geq 1, k \in \mathbb{Z})$

$$
H_{n+k}\left\{S^{n} ; M\right\}=\left(H_{n+k}\left(S^{n} ; M\right) \stackrel{H}{\longrightarrow} H_{n+k}\left(S^{n} \mid S^{n} ; M\right) \stackrel{P}{\longrightarrow} H_{n+k}\left(S^{n} ; M\right)\right) .
$$

For $k \geq 1$ this is a quadratic $\mathbb{Z}$-module as in (3.9). Using (5.11) we see

$$
H_{1+k}\left\{S^{1} ; M\right\}=\left\{\begin{aligned}
M, & k=0 \\
0, & \text { otherwise. }
\end{aligned}\right.
$$

Moreover by (7.10) we get for $n>1$

$$
H_{n+k}\left\{S^{n} ; M\right\}=\left\{\begin{array}{l}
0 \text { for } k<0 \text { or } k>n-1, \\
H_{k} M_{*}^{e} \text { for } 0 \leq k<n-1, \\
Z_{n-1} M_{*} \text { for } k=n-1 .
\end{array}\right.
$$

Hence only $H_{2 n-1}\left\{S^{n} ; M\right\}$ is quadratic and $H_{n+k}\left\{S^{n} ; M\right\}$ is an abelian group for $k \neq n-1$.

Proof of (7.10). For $k=0$ compare (6.11). For $k>0$ we have by the exact sequence (7.7) the formula, $X=M(A, n)$,

$$
H_{n+k}(X ; M)=H_{n+k}(X, \hat{M}) .
$$

If $A$ is free abelian the right hand side is computed in [11] by

$$
H_{n+k}(X, \hat{M})=\left(\bar{H}_{*} \otimes \hat{M}\right)_{n+k-1} .
$$

Here $\bar{H}_{*}=s^{-1} H_{*}(X, \mathbb{Z})$ is concentrated in degree $n-1$ so that the result in [11] yields the formulas of the proposition if $A$ is free abelian. If $A$ is not free abelian we use the exact sequence in (3.12).

(7.12) Remark. If $M$ is a quadratic $\mathbb{Z}$-module we can use (7.10) for the computation of square homology with coefficients in $M$. For this let $X \in$ space $_{1}$ and let

$$
X_{n}=M\left(A_{n}, n\right) \quad \text { with } \quad A_{n}=H_{n}(X, \mathbb{Z})
$$


be the Moore space of the $n t h$ homology of $X$. Then there exists a homotopy equivalence of chain complexes

$$
C_{*}(X) \simeq C_{*}\left(X_{1} \vee X_{2} \vee \ldots\right) .
$$

Hence we get by (7.4) an isomorphism

$$
\begin{aligned}
H_{n}(X ; M) & =H_{n}\left(X_{1} \vee X_{2} \vee \ldots ; M\right) \\
& =\bigoplus_{i \geq 1} H_{n}\left(X_{i} ; M\right) \oplus \bigoplus_{i<j} H_{n+1}\left(X_{i} \wedge X_{j} ; M_{e e}\right)
\end{aligned}
$$

In the second row we use the cross effect formula. Since we know $H_{n}\left(X_{i}\right)$ by $(7.10)$ we thus get a description for $H_{n}(X ; M)$ as an abelian group. For this we point out that for $A, B, M_{e e} \in \mathbf{A b}$

$$
H_{n}\left(M(A, i) \wedge M(B, j) ; M_{e e}\right)=\left\{\begin{array}{rll}
0 & \text { for } & n<i+j \text { and } n>i+j+2, \\
A \otimes B \otimes M_{e e} & \text { for } & n=i+j \\
\operatorname{Trp}\left(A, B, M_{e e}\right) & \text { for } & n=i+j+1 \\
A * B * M_{e e} & \text { for } & n=i+j+2 .
\end{array}\right.
$$

Here Trp is the triple torsion product of Mac Lane; see Notes on page 393 in [27]. Using (3) for $\hat{M}$ in (7.7) we thus obtain by the exact sequence in (7.7) a possibility to compute $H_{n}(X, M)$ for an arbitrary square group $M$ in terms of the boundary $\partial: H_{n}\left(X, M^{\text {add }}\right) \rightarrow H_{n-1}(X, \hat{M})$. For example we obtain the following vanishing theorem.

(7.13) Proposition. Let $X$ be a connected $C W$-space with $\operatorname{dim} X=N$ and let $M$ be a square group. Then

$$
H_{n}(X, M)=0 \quad \text { for } \quad n \geq 2 N
$$

and

$$
H_{n}(X, M)=H_{n}(X, \hat{M}) \quad \text { for } \quad N<n<2 N .
$$

\section{Stable square-homology and Steenrod squares}

Given a square group $M$ we obtain the square-homology $H_{n}(X ; M)$ and the stable square-homology $H_{n}^{S}(X ; M)$ which is the stabilization of $H_{n}(X ; M)$ as defined in (2.2). We have seen in (6.8) that the stable square-homology $H_{n}^{S}(X ; M)$ is a homology theory on space ${ }_{1}$. Hence by (2.8) there is a spectrum $E_{M}$ associated to the square group $M$ with

$$
H_{n}^{S}(X ; M)=H_{n}\left(X ; E_{M}\right) .
$$

This equation also holds if $X$ is not a finite $\mathrm{CW}$-space since both sides of the equation satisfy the colimit axiom.

(8.2) Remark. The correspondence $M \mapsto E_{M}$ yields a functor

$$
E: \text { Square } \rightarrow H o(\text { spectra })
$$

where the right hand side is the homotopy category of spectra. To obtain this functor we use Goodwillie's equivalence [17] between linear homotopy functors and 
spectra. Hence the functor $E$ is obtained by the functor which carries $M$ to $M_{\sharp}^{l i n}$ where the linear homotopy functor $M_{\sharp}^{l i n}$ determines functorially $E_{M}$ by

$$
M_{\sharp}^{\text {lin }}(X)=\Omega^{\infty}\left(E_{M} \wedge X\right) .
$$

Compare (4.3) and (4.11).

For an abelian group $A$ let $K(A)$ be the Eilenberg-Mac Lane spectrum with $K(A)_{n}=K(A, n)$. Let $K(A)[i]$ be the shifted spectrum with $K(A)[i]_{n}=$ $K(A, n+i)$ where $i \in \mathbb{Z}$. We clearly have

$$
H_{n}(X ; K(A)[i])=\tilde{H}_{n-i}(X ; A)
$$

where the right hand side is the reduced singular homology of $X$ with coefficients in $A$.

(8.4) Theorem. Let $M$ be a quadratic $\mathbb{Z}$-module. Then the stable square homology with coefficients in $M$ is given by an isomorphism, $X \in$ space $_{1}$,

$$
H_{n}^{S}(X, M)=\bigoplus_{i \geq 0} \tilde{H}_{n-i}\left(X, H_{i} M_{*}^{e}\right)
$$

where $M_{*}^{e}$ is the chain complex in (7.8). The isomorphism is natural in X. Hence by (8.3) the spectrum $E_{M}$ associated to $M$ is homotopy equivalent to the product of shifted Eilenberg-Mac Lane spectra $K\left(H_{i} M_{*}^{e}\right)[i]$ with $i \geq 0$.

Proof. We know by (4.11) that there exists a spectrum $E$ with

$$
M_{\sharp}^{\operatorname{lin}}(X)=\Omega^{\infty}(E \wedge X)
$$

Hence $E$ is determined by $M_{\sharp}^{\operatorname{lin}}\left(S^{n}\right)$ where we can use a large dimension $n$. Now in the stable range $M_{\sharp}\left(S^{n}\right) \rightarrow M_{\sharp}^{\operatorname{lin}}\left(S^{n}\right)$ is an equivalence. Here $M_{\sharp}\left(S^{n}\right)$ is given by an abelian simplicial group since $M$ is a quadratic $\mathbb{Z}$-module. An abelian simplicial group, however, is equivalent to a product of Eilenberg-Mac Lane spaces. Hence all $k$-invariants of $M_{\sharp}^{\text {lin }}\left(S^{n}\right)$ vanish and therefore $E$ is a product of Eilenberg-Mac Lane spectra.

(8.5) Theorem. Let $M$ be a square group and let $M^{\text {add }}=\operatorname{cok} P$ be defined by $M$. Then the spectrum $E_{M}$ associated to $M$ is homotopy equivalent to the cofiber of a map between spectra

$$
S q_{M}: K\left(M^{a d d}\right)[-1] \rightarrow \underset{i \geq 1}{\chi} K\left(H_{i} M_{*}^{e}\right)[i]
$$

Proof. For the proof we apply the Goodwillie calculus of functors which yields the fibre sequence in (4.11) (2). Applying this fiber sequence to $M_{\sharp}$ and $\hat{M}_{\sharp}$ in (7.7) 
gives us the rows in the following commutative diagram of homotopy functors.

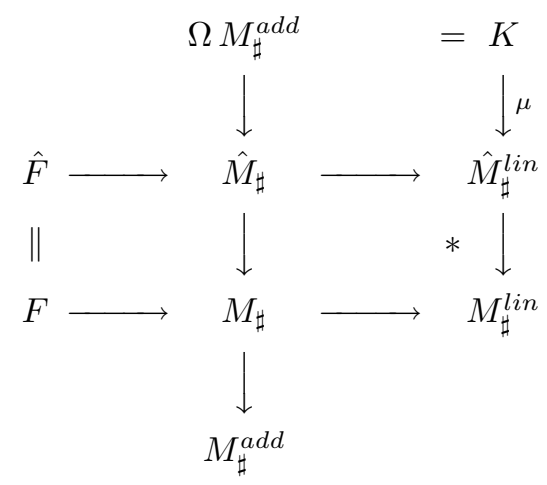

Here the column in the middle is obtained by the fiber sequence in (7.7). Now (4.11) (2) and (6.9) show that the fibres $F$ and $\hat{F}$ coincide so that the subdiagram * is a pull back. Hence the fiber $K$ of $\hat{M}_{\sharp}^{l i n} \rightarrow M_{\sharp}^{l i n}$ coincides with

$$
K=\Omega M_{\sharp}^{a d d}=K\left(M^{a d d}\right)[-1]_{\sharp}
$$

Here we denote by $E_{\sharp}$ the homotopy functor given by a spectrum $E$ with

$$
E_{\sharp}(X)=\Omega^{\infty}(E \wedge X) .
$$

Now $\mu$ in the diagram yields by (8.4) the map $S q_{M}$ in the theorem since a fiber sequence of spectra is also a cofiber sequence.

(8.6) Definition. The map $S q_{M}$ in (8.5) is a (multiple) cohomology operation which we call the squaring operation associated to the square group $M$. For $i \geq 1$ let

$$
S q_{M}^{i+1}: K\left(M^{a d d}\right)[-1] \rightarrow K\left(H_{i} M_{*}^{e}\right)[i]
$$

be the coordinate of $S q_{M}$ of degree $i+1$. Clearly $S q_{M}^{i+1}$ depends on the choice of the homotopy equivalence in (8.4). Below we show that the classical Steenrod squares

$$
S q^{i+1}: K(\mathbb{Z} / 2)[-1] \rightarrow K(\mathbb{Z} / 2)[i]
$$

yield examples of such squaring operations. Moreover we compute the operation $S q_{M}^{2}$ for any $M \in$ Square in the next section. Since in the bottom degree the homotopy equivalence in (8.4) is canonical we see that $S q_{M}^{2}$ is actually independent of the choice of the homotopy equivalence in (8.4). Hence $S q_{M}^{2}$ is also natural in $M$.

It is a classical result that any short exact sequence $A \longmapsto B \rightarrow C$ of abelian groups induces a long exact sequence of homology groups:

$$
\rightarrow H_{n}(X, A) \rightarrow H_{n}(X, B) \rightarrow H_{n}(X, C) \stackrel{\beta}{\rightarrow} H_{n-1}(X, A) \rightarrow
$$

where $\beta$ is the Bockstein operator. In a similar way one has for each short exact sequence $L \longmapsto M \rightarrow N$ of square groups a long exact sequence of square-homology groups:

$$
\rightarrow H_{n}(X, L) \rightarrow H_{n}(X, M) \rightarrow H_{n}(X, N) \stackrel{\beta}{\longrightarrow} H_{n-1}(X, L) \rightarrow .
$$


This sequence is obtained in the same way as the special case in (7.7) (2). In addition the stabilization of (8.7) yields the long exact sequence of stable squarehomology groups:

$$
\rightarrow H_{n}^{S}(X, L) \rightarrow H_{n}^{S}(X, M) \rightarrow H_{n}^{S}(X, N) \stackrel{\beta}{\longrightarrow} H_{n-1}^{S}(X, L) \rightarrow .
$$

Clearly the sequences (8.7) and (8.8) are natural with respect to maps between short exact sequences in Square. They are also natural in $X \in$ space $_{1}$. As a special case we obtain for $\hat{M} \longmapsto M \rightarrow M^{a d d}$ the commutative diagram:

$$
\begin{array}{ccc}
H_{n+1}^{S}\left(X, M^{\text {add }}\right) & \stackrel{\beta}{\longrightarrow} & H_{n}^{S}(X, \hat{M}) \\
\| & \| \\
\tilde{H}_{n+1}\left(X, M^{\text {add }}\right) \stackrel{\left(S q_{M}\right)_{*}}{\longrightarrow} & \bigoplus_{i \geq 1} \tilde{H}_{n-i}\left(X, H_{i} M_{*}^{e}\right)
\end{array}
$$

Here the bottom arrow is induced by the squaring operation $S q_{M}$ in (8.6). For this recall that a map $\phi: E \rightarrow E^{\prime}$ between spectra induces a map $\phi_{*}: H_{n}(X, E) \rightarrow$ $H_{n}\left(X, E^{\prime}\right)$ between homology groups. See (2.8) and (8.3). In diagram (8.9) the homomorphism $\beta$ is natural in $X$ and $M$. The isomorphism on the right hand side of (8.9) is given by the choice in (8.4).

It is well known that the Steenrod square $S q^{1}$ is obtained as a Bockstein operator; that is,

$$
\beta=\left(S q^{1}\right)_{*}: H_{n+1}(X, \mathbb{Z} / 2) \rightarrow H_{n}(X, \mathbb{Z} / 2)
$$

coincides with the Bockstein operator associated to $\mathbb{Z} / 2 \longmapsto \mathbb{Z} / 4 \rightarrow \mathbb{Z} / 2$. The next result shows that all Steenrod squares $S q^{i}, i \geq 1$, are actually obtained by a Bockstein operator of stable square-homology. For this we use the square group

$$
\mathbb{Z}_{\text {nil }}^{4,2}=(\mathbb{Z} / 4 \stackrel{H}{\longrightarrow} \mathbb{Z} / 2 \stackrel{0}{\longrightarrow} \mathbb{Z} / 4)
$$

with $H\{n\}=\{n(n-1) / 2\}$. Here $\{n\} \in \mathbb{Z} / k$ denotes the coset of $n \in \mathbb{Z}$. For $\mathbb{Z}_{n i l}$ in (6.3) one has a canonical quotient map $q: \mathbb{Z}_{\text {nil }} \rightarrow \mathbb{Z}_{\text {nil }}^{4,2}$ given by $\mathbb{Z} \rightarrow \mathbb{Z} / 4$ and $\mathbb{Z} \rightarrow \mathbb{Z} / 2$. There is a short exact sequence in Square

$$
(\mathbb{Z} / 2)^{\Gamma} \longmapsto \mathbb{Z}_{\text {nil }}^{4,2} \rightarrow \mathbb{Z} / 2
$$

given by the diagram

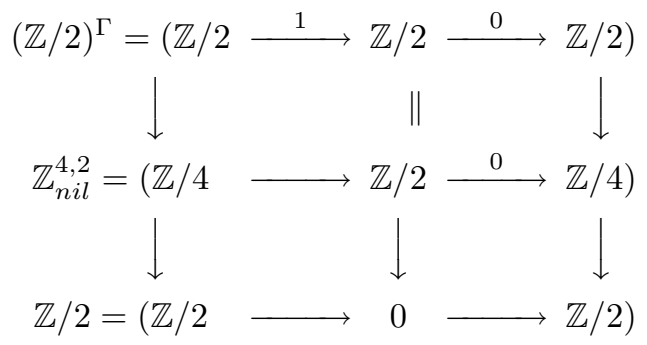

One readily checks that for $M=(\mathbb{Z} / 2)^{\Gamma}$ one has $H_{i} M_{*}^{e}=\mathbb{Z} / 2$ for $i \geq 0$. Hence one gets by (8.4) a Bockstein operator $\beta$ as in the following theorem. 
(8.13) Theorem. Let $\beta$ be the Bockstein operator of stable square homology associated to the short exact sequence in (8.12). Then the following diagram commutes:

$$
\begin{array}{ccc}
H_{n+1}^{S}(X, \mathbb{Z} / 2) \stackrel{\beta}{\longrightarrow} & H_{n}^{S}\left(X,(\mathbb{Z} / 2)^{\Gamma}\right) \\
\| & \| \\
\tilde{H}_{n+1}(X, \mathbb{Z} / 2) \stackrel{S q}{\longrightarrow} \underset{i \geq 0}{\oplus} \tilde{H}_{n-i}(X, \mathbb{Z} / 2)
\end{array}
$$

Here $S q$ has the coordinates $\left(\chi S q^{i+1}\right)_{*}$ induced by the Steenrod squares $S q^{i+1}$ for $i \geq 0$ where $\chi$ is the anti-automorphism of the Steenrod algebra; see 27.24 [22]. Moreover the isomorphism on the right hand side of the diagram is obtained by an appropriate choice of the homotopy equivalence in (8.4).

For the proof of this theorem we use the mod-2 restricted lower central series $\bar{\Gamma}_{n}(G)$ which defines the group functor

$$
\overline{n i l}_{2}: \mathbf{G r} \rightarrow \mathbf{G r} \quad \text { with } \quad \overline{n i l}_{2}(G)=G / \bar{\Gamma}_{3}(G)
$$

This is the restricted version of $n i l_{2}$ in (5.11). For a free group $G$ we have the following facts (1), (2) and (3).

$$
G / \bar{\Gamma}_{2}(G)=G^{a b} \otimes \mathbb{Z} / 2=V
$$

is a $\mathbb{Z} / 2$-vector space and

$$
\bar{\Gamma}_{2}(G) / \bar{\Gamma}_{3}(G)=\bar{L}_{2}(V) .
$$

Here $\bar{L}_{2}(V) \subset V \otimes V$ is generated by $[v, w]=v \otimes w+w \otimes v$ and $v \otimes v$ for $v, w \in V$. $\bar{L}_{2}(V)$ is the degree 2 part of the free mod-2 restricted Lie algebra $\bar{L}(V)$. Using $7.6[15]$ one obtains (1) and (2) above. Hence one obtains the natural short exact sequence in the top row of the following commutative diagram. The bottom row is obtained by the exact sequence in (8.12) and the quadratic tensor product.

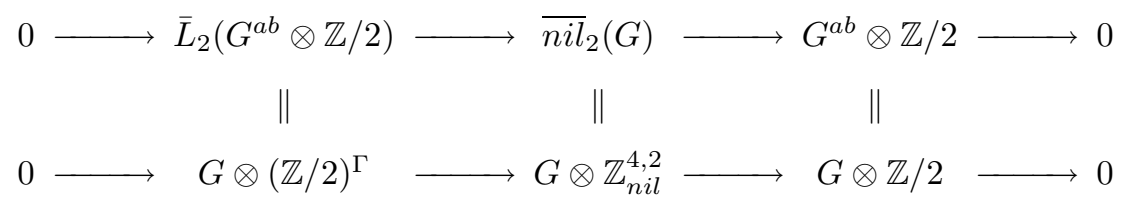

The vertical isomorphisms which are natural in $G$ are induced by $G \otimes \mathbb{Z}_{n i l}=n i l_{2}(G)$ in (6.4); see 8.1 [10].

Proof of (8.13). If $G=\alpha(X)$ is the free simplicial group given by $X$ then the exact sequence of simplicial groups in (8.14) (3) induces the Bockstein operator in (8.13). The connecting homomorphism

$$
d_{1}: \pi_{n}\left(G^{a b} \otimes \mathbb{Z} / 2\right) \rightarrow \pi_{n-1} \bar{L}_{2}\left(G^{a b} \otimes \mathbb{Z} / 2\right)
$$

is computed in 8.10 [15] in terms of the Steenrod operations $S q^{i}$ which act from the right on homology since for a finite type space $X$ we have $H_{*}(X, \mathbb{Z} / 2)=$ $\operatorname{Hom}\left(H^{*}(X, \mathbb{Z} / 2), \mathbb{Z} / 2\right)$. The anti-isomorphism $\chi$ of the Steenrod algebra has the property that for $y \in H_{*}(X, \mathbb{Z} / 2)$ one has

$$
\left(\chi S q^{i}\right)_{*}(y)=y S q^{i}
$$

Therefore the stabilization of the differential $d_{1}$ in 8.10 [15] yields the result. 
As an application of (8.13) and (8.5) we obtain the following results which determine explicitly the spectra $E_{M}$ associated to $M=\mathbb{Z}_{n i l}$ and $M=\mathbb{Z}_{\text {nil }}^{4,2}$ respectively. For this we consider the following commutative diagram in Square:

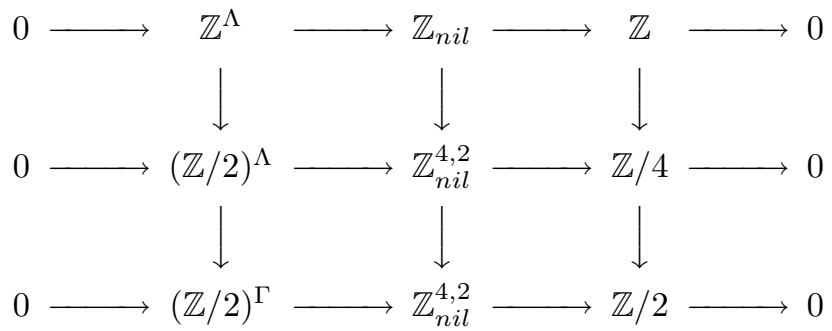

Here the rows are exact and the vertical arrows are quotient maps. The top row and the row in the middle are the exact sequences $\hat{M} \longmapsto M \rightarrow M^{\text {add }}$ for $M=\mathbb{Z}_{\text {nil }}$ and $M=\mathbb{Z}_{\text {nil }}^{4,2}$ respectively. We have $\mathbb{Z}^{\Lambda}=(0 \rightarrow \mathbb{Z} \rightarrow 0)$ and $(\mathbb{Z} / 2)^{\Lambda}=(0 \rightarrow \mathbb{Z} / 2 \rightarrow 0)$. Naturality of the Bockstein operator yields by (8.9) a relation of $S q_{M}$ for $M=\mathbb{Z}_{\text {nil }}$ and $M=\mathbb{Z}_{\text {nil }}^{4,2}$ with $\beta$ in (8.13). Since the maps $\mathbb{Z}^{\Lambda} \rightarrow(\mathbb{Z} / 2)^{\Lambda} \rightarrow(\mathbb{Z} / 2)^{\Gamma}$ induce injections

$$
H_{*}\left(\mathbb{Z}^{\Lambda}\right)_{*}^{e} \longmapsto H_{*}\left(\mathbb{Z} / 2^{\Lambda}\right)_{*}^{e} \longmapsto H_{*}\left(\mathbb{Z} / 2^{\Gamma}\right)_{*}^{e}
$$

(see $(7.8)$ ) we get the following results.

(8.16) Theorem. For the square group $M=\mathbb{Z}_{\text {nil }}$ we have the spectrum $E=E_{M}$ with

$$
H_{n}^{S}\left(X, n i l_{2}\right)=H_{n}^{S}\left(X, \mathbb{Z}_{n i l}\right)=H_{n}(X, E) .
$$

This spectrum $E$ is homotopy equivalent to the cofiber of the map

$$
S q_{M}: K(\mathbb{Z})[-1] \rightarrow \underset{i \text { odd }}{\times} K(\mathbb{Z} / 2)[i] .
$$

Here the coordinate of degree $i+1$ is $q^{*}\left(\chi S q^{i+1}\right)$ where $q: \mathbb{Z} \rightarrow \mathbb{Z} / 2$ is the quotient map and $\chi$ is the anti-automorphism of the Steenrod algebra.

(8.17) Theorem. For the square group $M=\mathbb{Z}_{\text {nil }}^{4,2}$ we have the spectrum $E=E_{M}$ with

$$
H_{m}^{S}\left(X, \overline{n i l}_{2}\right)=H_{n}^{S}\left(X, \mathbb{Z}_{n i l}^{4,2}\right)=H_{n}(X, E) .
$$

This spectrum $E$ is homotopy equivalent to the cofiber of the map

$$
S q_{M}: K(\mathbb{Z} / 4)[-1] \rightarrow \underset{i \geq 1}{\times} K(\mathbb{Z} / 2)[i] .
$$

Here the coordinate of degree $i+1$ is $q^{*}\left(\chi S q^{i+1}\right)$ where $q: \mathbb{Z} / 4 \rightarrow \mathbb{Z} / 2$ is the quotient map and $\chi$ is the anti-automorphism of the Steenrod algebra.

\section{The SQUARING Operation $S q_{M}^{2}$}

The squaring operation $S q_{M}^{2}$ associated to a square group $M$ is an element in the following group where $n$ is large; see (8.6).

$$
\begin{aligned}
& {\left[K\left(M^{\text {add }}\right)[-1], K\left(H_{1} M_{*}^{e}\right)[1]\right)} \\
& \quad=\left[K\left(M^{a d d}, n-1\right), K\left(H_{1} M_{*}^{e}, n+1\right)\right] \\
& \quad=\operatorname{Hom}\left(M^{\text {add }} \otimes \mathbb{Z} / 2, H_{1} M_{*}^{e}\right) .
\end{aligned}
$$


Here we have $M^{\text {add }}=\operatorname{cok}(P)$ and $H_{1} M_{*}^{e}=\operatorname{ker}(P) / \operatorname{im}(2-H P)$ so that $S q_{M}^{2}$ is a homomophism

$$
S q_{M}^{2}: \operatorname{cok}(P) \otimes \mathbb{Z} / 2 \rightarrow \operatorname{ker}(P) / \operatorname{im}(2-H P) .
$$

We now consider the following diagram where $(\mid)_{H}$ is the cross effect of $H$ in (6.3) and where $\Delta$ is the diagonal with $\Delta(x)=(x, x)$ for $x \in M_{e}$.

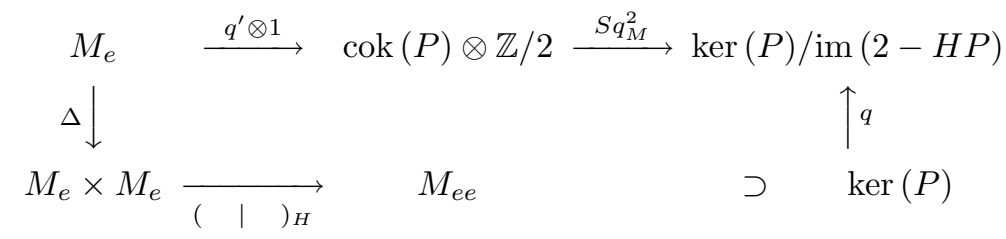

Here $q$ is the quotient map and $q^{\prime}: M_{e} \rightarrow \operatorname{cok}(P)$ is the quotient map.

(9.3) Theorem. For a square group $M$ there is a unique homomoprhism $S q_{M}^{2}$ such that diagram (9.2) commutes and this homomorphism coincides with the squaring operation $S q_{M}^{2}$ by use of (9.1).

Proof of (9.3). There exist in fact only two homomorphisms from $M_{e}$ to $\operatorname{ker}(P) /$ $\operatorname{im}(2-H P)$ which are defined for all square groups $M$ and which are natural in $M$. We have seen that $S q_{M}^{2}$ in (8.6) is natural in $M$. Moreover $S q_{M}^{2}$ in (8.6) is nontrivial by (8.16). But the nontrivial natural map is $S q_{M}^{2}\left(q^{\prime} \otimes 1\right)$ in (9.2) so that $S q_{M}^{2}$ has to coincide with $S q_{M}^{2}$ in (9.2).

For example for $M=\mathbb{Z}_{n i l}$ the homomorphism $S q_{M}^{2}$ is the isomorphism $S q_{M}^{2}$ : $\mathbb{Z} \otimes \mathbb{Z} / 2 \cong \mathbb{Z} / 2$. We now give an interpretation of diagram (9.2) in terms of algebraic models of $(n-1)$-connected $(n+1)$-types, $n \geq 2$, obtained in [6]. This leads to an additional proof of (9.3).

(9.4) Definition. A reduced quadratic module $(\omega, \delta)$ is a diagram

$$
M^{a b} \otimes M^{a b} \stackrel{\omega}{\longrightarrow} L \stackrel{\delta}{\longrightarrow} M
$$

of homomorphisms between groups such that the following properties hold. The groups $M, L$ have nilpotency degree 2 and the quotient map $M \rightarrow M^{a b}$ is denoted by $x \mapsto\{x\}$. Then for $x, y \in L, a, b \in M$ we have

$$
\begin{aligned}
& \delta \omega(\{a\} \otimes\{b\})=-a-b+a+b, \\
& \omega(\{\delta x\} \otimes\{\delta y\})=-x-y+x+y, \\
& \omega(\{\delta x\} \otimes\{a\}+\{a\} \otimes\{\delta x\})=0 .
\end{aligned}
$$

We say that $(\omega, \delta)$ is a stable quadratic module if in addition

$$
\omega(\{a\} \otimes\{b\}+\{a\} \otimes\{b\})=0
$$

A morphism $(\omega, \delta) \rightarrow\left(\omega^{\prime}, \delta^{\prime}\right)$ is a commutative diagram in $\mathbf{G r}$

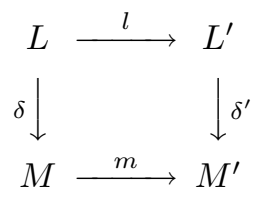


such that $l \omega=\omega^{\prime}\left(m^{a b} \otimes m^{a b}\right)$. This is a weak equivalence if $(l, m)$ induces isomorphisms

$$
\operatorname{ker}(\delta) \cong \operatorname{ker}\left(\delta^{\prime}\right), \operatorname{cok}(\delta) \cong \operatorname{cok}\left(\delta^{\prime}\right) .
$$

Let rquad (resp. squad) be the categories of reduced (resp. stable) quadratic modules and let Horquad, Hosquad be the localization with respect to weak equivalences. There are equivalences of categories for which the following diagram commutes, $n \geq 3$; see [6] and [8].

$$
\begin{array}{ccc}
\operatorname{types}_{2}^{1} & \stackrel{\lambda_{2}}{\longrightarrow} & \text { Horquad } \\
\Omega^{n-2} \cup & & \cup i \\
\operatorname{types}_{n}^{1} & \stackrel{\lambda_{n}}{\longrightarrow} & \text { Hosquad }
\end{array}
$$

Here $\operatorname{types}_{k}^{1}$ is the homotopy catgeory of CW-spaces $X$ for which $\pi_{i} X=0$ for $i<k$ and $i>k+1$. Moreover $\Omega^{n-2}$ is the iterated loop space functor and $i$ is the inclusion functor. For $X \in \operatorname{types}_{n}^{1}$ and $(\omega, \delta)=\lambda_{n}(X)$ we have natural isomorphisms

$$
\pi_{n} X=\pi_{n}=\operatorname{cok}(\delta), \pi_{n+1}(X)=\pi_{n+1}=\operatorname{ker}(\delta) .
$$

Moreover the $k$-invariant of $X$ is an element

$$
k(X) \in H^{n+2}\left(K\left(\pi_{n}, n\right), \pi_{n+1}\right)=\operatorname{Hom}\left(\Gamma_{n}^{1}\left(\pi_{n}\right), \pi_{n+1}\right) .
$$

Here $\Gamma_{n}^{1}$ is the functor $\mathbf{A b} \rightarrow \mathbf{A b}$ which is Whitehead quadratic functor $\Gamma$ for $n=2$ and $\otimes \mathbb{Z} / 2$ for $n \geq 3$. We can obtain $k(X)$ from $(\omega, \delta)=\lambda_{n}(X)$ as follows. Given $(\omega, \delta) \in$ rquad there is a unique homomorphism $k$ for which the following diagram commutes:

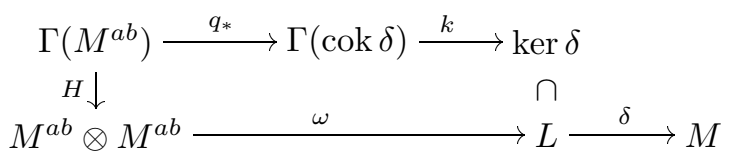

Here $H$ is the cross effect map in (3.1) for the functor $\Gamma$ and $q_{*}$ is induced by the quotient map $M \rightarrow M^{a b} \rightarrow \operatorname{cok} \delta$ which factors through $M^{a b}$. If $(\omega, \delta)$ is stable then $k$ admits a factorization

$$
k: \Gamma(\operatorname{cok} \delta) \stackrel{\sigma}{\longrightarrow} \operatorname{cok}(\delta) \otimes \mathbb{Z} / 2 \stackrel{k^{\prime}}{\longrightarrow} \operatorname{ker} \delta .
$$

Now the $k$-invariant $k(X)$ with $(\omega, \delta)=\lambda_{n} X$ is $k$ for $n=2$ and is $k^{\prime}$ for $n \geq 3$. Compare $[6,8]$.

Recall that Square is the category of square groups in (6.3). We now define canonical algebraic functors

$$
\text { Square } \stackrel{\tau}{\longrightarrow} \text { rquad } \stackrel{L}{\longrightarrow} \text { Ho rquad }
$$

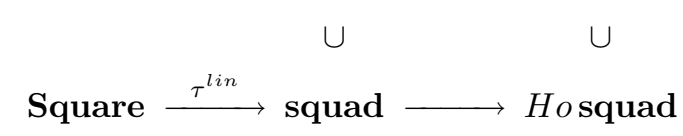


together with a natural transformation $a: \tau \rightarrow \tau^{l i n}$. Here $L$ is the localization functor. The functor $\tau$ is given by

$$
\tau(M)=\left(M_{e}^{a b} \otimes M_{e}^{a b} \stackrel{\omega}{\longrightarrow} M_{e e} \stackrel{\delta}{\longrightarrow} M_{e}\right)
$$

where $\omega(\{x\} \otimes\{y\})=(x \mid y)_{H}$ and $\delta=P$. Moreover let

$$
\tau^{l i n}(M)=\left(M_{e}^{a b} \otimes M_{e}^{a b} \stackrel{\omega^{\prime}}{\longrightarrow} M_{e e} / i m(2-H P) \stackrel{\delta^{\prime}}{\longrightarrow} M_{e}\right)
$$

where $\omega^{\prime}$, resp. $\delta^{\prime}$, are induced by $\omega$, resp. $\delta$, in $\tau(M)$ above. We clearly have the natural map $a: \tau(M) \rightarrow \tau^{l i n}(M)$ which is the identity on $M_{e}$ and the quotient map on $M_{e e}$. One readily checks that the functors $\tau$ and $\tau^{l i n}$ are well defined; for this we only prove

(9.7) Lemma. $\tau^{\text {lin }}(M)$ is a stable quadratic module.

Proof. We have to show that for $a, b \in M_{e}$ we have $(a \mid b)_{H}+(b \mid a)_{H} \in \operatorname{im}(2-H P)$. But we know by 3.5 (4) in [10] that $\Delta: M_{e} \rightarrow M_{e e}$ with

$$
\Delta(a)=(H P-2) H a+(a \mid a)_{H}
$$

is a homomorphism. Hence we get

$$
\begin{aligned}
& (a \mid b)_{H}+(b \mid a)_{H}=(a+b \mid a+b)_{H}-(a \mid a)_{H}-(b \mid b)_{H} \\
= & \Delta(a+b)+(2-H P) H(a+b) \\
& -(\Delta(a)+(2-H P) H(a)+\Delta(b)+(2-H P) H(b)) \\
= & (2-H P)(a \mid b)_{H} .
\end{aligned}
$$

This term needs not to be trivial in $M_{e e}$ as the example $\mathbb{Z}_{n i l}$ shows. Hence $\tau(M)$ in general is not stable.

We now obtain functors

$$
S, S^{l i n}: \text { Square } \rightarrow \operatorname{types}_{2}^{1}
$$

which carry the square group $M$ to $S(M)=M_{\sharp}\left(S^{2}\right)$ and $S^{l i n}(M)=3$-type of $M_{\sharp}^{l i n}\left(S^{2}\right)$ respectively. Here (7.10) shows that $M_{\sharp}\left(S^{2}\right) \in \operatorname{types}_{2}^{1}$. We have the obvious map

$$
b: S(M)=M_{\sharp}\left(S^{2}\right) \rightarrow M_{\sharp}^{l i n}\left(S^{2}\right) \rightarrow S^{l i n}(M)
$$

which is natural in $M$. We also observe that $S^{l i n}(M)$ is an infinite loop space since $M_{\sharp}^{\text {lin }}\left(S^{2}\right)$ is an infinite loop space.

(9.9) Theorem. For the functors in (9.8), (9.6) and (9.5) there exist natural isomorphisms such that the following diagram commutes.

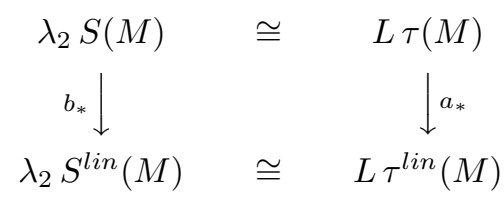

Proof of (9.3). One readily checks that $S q_{M}^{2}$ in (9.2) is the $k$-invariant of $\tau^{l i n}(M)$. The $k$-invariant of $\tau^{l i n}(M)$ coincides with the first non-trivial $k$-invariant of $M_{\sharp}^{\text {lin }}\left(S^{2}\right)$ by (9.9). This yields the proposition in (9.3). 
Proof of (9.9). We have by (7.10) that

$$
\begin{aligned}
& \pi_{2}(S(M))=\pi_{2} M^{\sharp}\left(S^{2}\right)=\mathbb{Z} \otimes \operatorname{cok}(P)=\operatorname{cok}(P), \\
& \pi_{3}(S(M))=\pi_{3} M^{\sharp}\left(S^{2}\right)=\mathbb{Z} \otimes Z_{1} M_{*}=\operatorname{ker}(P) .
\end{aligned}
$$

Hence the isomorphism $\lambda_{2} S(M) \cong L \tau(M)$ exists on the level of homotopy groups. An explicit isomorphism is given by the result of Conduché [14]. For this let $G$ be the free simplicial group generated by one element in degree 1 . Then we have $G \simeq \alpha\left(S^{2}\right)$ in (5.1) so that $M^{\sharp}\left(S^{2}\right) \simeq B|G \otimes M|$. Here $G \otimes M$ is a simplicial group with only two nontrivial homotopy groups $\pi_{1}$ and $\pi_{2}$. Hence the homotopy type of $G \otimes M$ is described by its reduced 2-module in the sense of Conduché (see (2.9) [14]). But this reduced 2-module of $G \otimes M$ coincides with $\tau(M)$. This yields the isomorphism $\lambda_{2} S(M) \cong L \tau(M)$. Moreover we know that $a_{*}$ coincides with $b_{*}$ on the level of homotopy groups since

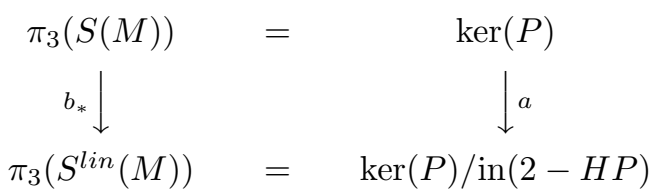

commutes. This shows that the $k$-invariant of $S^{\operatorname{lin}}(M)$ is actually the $k$-invariant of $\tau^{l i n}(M)$. Moreover the natural isomorphism $\lambda_{2} S(M) \cong L \tau(M)$ induces the natural isomorphism $\lambda_{2} S^{l i n}(M) \cong L \tau^{l i n}(M)$.

\section{ApPENDIX A: A CRITERION FOR 2-EXCISIVE FUnCTORS}

We describe a criterion for 2-excisive functors which shows that only very special strongly co-Cartesian diagrams are needed to determine a 2-excisive functors. We use this result for the proof of theorem (6.6). It would be interesting to obtain a similar result for $n$-excisive functors.

Let $\underline{Y}$ be a 2-cube consisting of spaces $Y_{1}, Y_{2}, Y_{3}, Y_{4}$ and let $A \in$ space. Then we define $A \vee \underline{Y}$ by

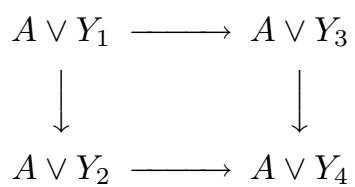

This yields the 3 -cube $(0,1): A \vee \underline{Y} \rightarrow \underline{Y}$. Clearly if $\underline{Y}$ is co-Cartesian then $(0,1): A \cup \underline{Y} \rightarrow \underline{Y}$ is a strongly co-Cartesian 3-cube. Recall the definition of Cube $(X, A)$ in the proof of (4.12).

(A.1) Theorem. Let $D:$ space $_{r} \rightarrow$ space $_{1}, r \geq 0$, be a homotopy functor. Assume that for pairs $(X, A) \in$ pair $_{r}$ and all homotopy push outs $\underline{Y}$ in space $_{r}$ the 3-cubes $D(C u b e(X, A))$ and $D((0,1): A \vee \underline{Y} \rightarrow \underline{Y})$ are Cartesian. Then the homotopy functor $D$ is 2-excisive.

The theorem shows that essentially the 3 -cubes $C u b e(X, A)$ suffice to determine quadratic homotopy functors. This again shows that the quadratic excision sequence essentially covers all excision properties of a 2-excisive functor.

Proof of (6.6). Let $F$ be a quadratic coefficient functor so that $F=\otimes M$ by (6.4). Since we can use (5.4) it is enough to prove that $F_{\sharp}$ is 2 -excisive. For this we can use theorem (A.1) above. Using (1) in the proof of (6.9) above we see that 
$F_{\sharp}((0,1): A \vee \underline{Y} \rightarrow \underline{Y})$ is Cartesian if $\underline{Y}$ is co-Cartesian. Moreover using the pull back diagram (4) in the proof of $(6.9)$ we see that $F_{\sharp}\left(\mathrm{Cube}\left(X^{\prime}, A^{\prime}\right)\right)$ is Cartesian for $\left(X^{\prime}, A^{\prime}\right) \in$ pair $_{1}$. For this we use (4.8). Hence the assumptions of (A.1) are satisfied and therefore $F_{\sharp}$ is 2 -excisive.

Proof of (A.1). Let $\underline{Y}$ be a cofibrant co-Cartesian 2-cube and let $i: \underline{Y} \subset A \vee \underline{Y}$ be the inclusion then $D(i)$ is Cartesian since $D(0,1)$ in $(6.8)$ is Cartesian and $(0,1) i=1$. For this we use the lemmas in section 1 of [12]. Next let $\left(Y_{1}, A\right)$ be a pair in pair ${ }_{r}$. Then we obtain the 3-cube $q: \underline{Y} \rightarrow \underline{Y} / A$ given by the quotient maps $q_{i}: Y_{i} \rightarrow Y_{i} / A$. Clearly $q$ is strongly co-Cartesian. For this we put the cubes Cube $\left(Y_{i}, A\right)$ and the cube $(0,1): A \vee \underline{Y} \rightarrow \underline{Y}$ together to form a large cube with boundary $q: \underline{Y} \rightarrow \underline{Y} / A$. We apply $D$ to this large cube and then we take homotopy fibers which form the following commutative diagram where $Q_{i}$ is the homotopy fiber of $\left(q_{i}\right)_{*}: D\left(Y_{i}\right) \rightarrow D\left(Y_{i} / A\right)$.

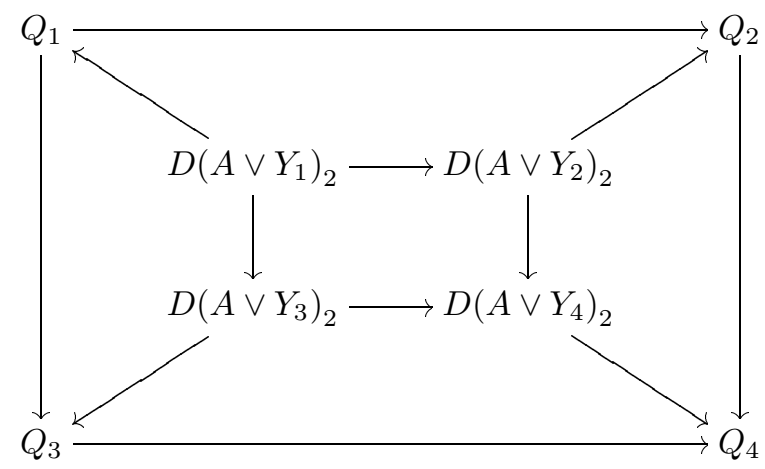

Here the inside square is Cartesian since $D(0,1)$ is Cartesian by the assumption in (A.1). Moreover, since $D\left(C u b e\left(Y_{i}, A\right)\right)$ is Cartesian by the assumption in (A.1) we see that the four boundary subdiagrams are Cartesian by (4.8). For this we use the following diagram; compare the proof of (4.9).

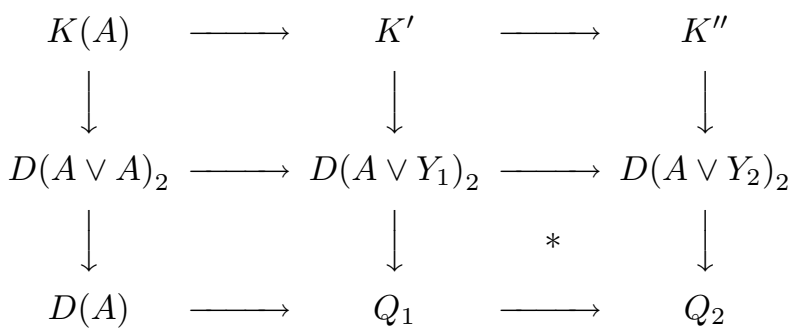

Here the columns are fiber sequences. We know that $K(A) \rightarrow K^{\prime}$ and $K(A) \rightarrow K^{\prime \prime}$ are homotopy equivalences. Hence also $K^{\prime} \rightarrow K^{\prime \prime}$ is a homotopy equivalence and therefore the subdiagram * is Cartesian. Now all subdiagrams of (1) being Cartesian we see that also the boundary diagram of (1) is Cartesian. This completes by (4.8) the proof that $D(q: \underline{Y} \rightarrow Y / A)$ is Cartesian. Now let $\underline{Y} \cup C A$ be the 2-cube given by $Y_{i} \cup_{A} C A$. Since the quotient map $Y_{i} \cup C A \rightarrow Y_{i} / A$ is a homotopy equivalence we see that also the inclusion $j: \underline{Y} \subset \underline{Y} \cup C A$ is a 3-cube for which $D(j)$ is Cartesian. Now let $\underline{X}$ and $\underline{Y}$ be 2-cubes and let $f: \underline{Y} \rightarrow \underline{X}$ be a strongly co-Cartesian 3cube. We may assume up to equivalence that $f$ is cofibrant, that is all maps in the 3-cube $f$ are cofibrations and all subsquares are actually push outs. Using 
CW-decomposition we can filter $f$ by an infinite sequence

$$
f: \underline{Y}=\underline{X}_{0} \subset \underline{X}_{1} \subset \underline{X}_{2} \subset \ldots \subset \operatorname{colim}\left(\underline{X}_{i}\right) \simeq \underline{X} .
$$

Here all $\underline{X}_{i} \subset \underline{X}_{i+1}$ are cofibrant co-Cartesian 3-cubes for which there exist $A_{i} \in$ space $_{r}$ such that $\underline{X}_{1}=\underline{Y} \vee A_{1}$ and $X_{i+1}=X_{i} \cup C A_{i}, i \geq 1$. We have seen above that $D\left(\underline{X}_{i} \rightarrow \underline{X}_{i+1}\right)$ is Cartesian for all $i \geq 0$. This implies by I.4b.5 in [3] that also $D(\underline{Y} \rightarrow \underline{X})$ is Cartesian since we assume that $D$ preserves filtered colimits.

\section{Appendix B: The homology SPECTRAL SEQUence}

Let $D$ be a reduced homotopy functor. We describe a spectral sequence which converges to the homology with coefficients in $D$. If $D$ is linear this is the AtiyahHirzebruch spectral sequence.

(B.1) Definition. Let $D:$ space $_{1} \rightarrow$ space $_{1}$ be a reduced homotopy functor and let $X$ be a connected CW-complex. Let $U=C(X)$ be the reduced cone on $X$ which is filtered by $X=U^{0} \subset U^{1} \subset \ldots \subset U$ with $U^{n}=X \cup C\left(X^{n-1}\right), n \geq 1$. For $(Y, B) \in$ pair $_{1}$ let

$$
H_{n}(Y, B)=H_{n}(Y, B ; D)
$$

be the $D$-homology in (4.2). Then the filtered space $U$ gives rise to the following spectral sequence. First we can form the long exact sequence

$$
\begin{aligned}
\ldots \rightarrow H_{2}\left(U^{n}\right) & \stackrel{j}{\longrightarrow} H_{2}\left(U^{n}, U^{n-1}\right) \stackrel{\partial}{\longrightarrow} H_{1}\left(U^{n-1}\right) \\
& \stackrel{i}{\longrightarrow} H_{1}\left(U^{n}\right) \stackrel{j}{\longrightarrow} H_{1}\left(U^{n}, U^{n-1}\right) \rightarrow 0
\end{aligned}
$$

where the last object is a set with basepoint 0 and all the other objects are groups. We can form the rth derived homotopy sequences of (2) for $r \geq 0, n \leq 0$,

$$
\begin{aligned}
\ldots \rightarrow H_{2}^{(r)}(n-2 r-1) & \rightarrow F_{1}^{(r)}(n-r) \rightarrow H_{1}^{(r)}(n-r) \\
& \rightarrow H_{1}^{(r)}(n-r-1) \rightarrow F_{0}^{(r)}(n) \rightarrow 0 .
\end{aligned}
$$

Here we set for $q \in \mathbb{Z}, n=-m$,

$$
\begin{aligned}
H_{q}^{(r)}(n)= & \operatorname{image}\left(H_{q}\left(U^{m-r}\right) \rightarrow H_{q}\left(U^{m}\right)\right), \\
F_{q}^{(r)}(n)= & \operatorname{kernel}\left(H_{q+1}\left(U^{m+1}, U^{m}\right) \rightarrow H_{q}\left(U^{m}\right) / H_{q}^{(r)}(n)\right) / \\
& \quad \text { action of } \operatorname{kernel}\left(H_{q+1}\left(U^{m+1}\right) \rightarrow H_{q+1}\left(U^{m+r+1}\right)\right) .
\end{aligned}
$$

For $r=0$ the sequence (3) coincides with (2). One can check that (3) is well defined and has the same properties as (2). Let

$$
E_{r}^{s, t}=F_{t-s}^{(r-1)}(s) \quad \text { for } \quad t \in \mathbb{Z}, s \leq 0, r \geq 1
$$

and let the differential $d_{r}: E_{r}^{s, t} \rightarrow E_{r}^{s+r, t+r-1}$ of degree $(r, r-1)$ be the composition

$$
F_{(t-s)}^{(r-1)}(s) \rightarrow H_{t-s}^{(r-1)}(s) \rightarrow F_{t-s-1}^{(r-1)}(s+r)
$$

where we use the operators from (3). Clearly we have for $q \geq 0, s \geq 0$

$$
E_{1}^{-s, q-s}=F_{q}^{(0)}(-s)=H_{q+1}\left(U^{s+1}, U^{s}\right)
$$

and $d_{1}$ is the composition

$$
d_{1}=j \partial: H_{q+1}\left(U^{s+1}, U^{s}\right) \rightarrow H_{q}\left(U^{s}\right) \rightarrow H_{q}\left(U^{s}, U^{s-1}\right) .
$$


Assume that the pairs $\left(U^{m}, U^{m-1}\right)$ have the property that there exist $0 \leq N_{0} \leq$ $N_{1} \leq \ldots$ with $\lim \left\{N_{m}\right\}=\infty$ such that

$$
H_{i}\left(U^{m+1}, U^{m}\right)=0 \quad \text { for } \quad i<N_{m} .
$$

Then we can find for each $q \geq 0$ a bound $r=r(q)<\infty$ such that

$$
E_{r}^{s, q+s}=E_{r+1}^{s, q+1}=\ldots=E_{\infty}^{s, q+s} .
$$

We obtain a filtration of $H_{q}(X)$ for $q \geq 1, s \geq 0$,

$$
\begin{aligned}
& 0=K_{0, q} \subset K_{1, q} \subset \ldots \subset K_{s, q} \subset \ldots \subset H_{q}(X), \\
& K_{s, q}=\operatorname{kernel}\left(H_{q}(X) \rightarrow H_{q}\left(X / X^{s}\right)\right) .
\end{aligned}
$$

Since $D$ is reduced we see that $\bigcup_{s \geq 0} K_{s, q}=H_{q}(X)$ and

$$
E_{\infty}^{-s, q-s}=K_{s, q} / K_{s+1, q} .
$$

A similar spectral sequence is available for reduced homotopy functors which map to spectra; compare (4.1).

(B.2) Remark. The spectral sequence $E_{r}^{s, t}$ above coincides with the spectral sequence in (III.10.4) [3] by considering the relative homotopy groups of the filtered space $\left\{D\left(U^{n}\right)\right\}$. The conventions for indexing $E_{r}^{s, t}$ arises from the comparison with the Bousfield-Kan spectral sequence [12]; compare the discussion in (III.10.2) [3]. We can alter the indexing by defining

$$
E_{s, t}^{r}=E_{r}^{-s, t}, \quad s \geq 0, t \in \mathbb{Z} .
$$

Then the differential for $E_{s, t}^{r}$ has degree $(-r, r-1)$ as in the spectral sequence discussed by G.W. Whithead, chapter XIII, page 614 [29]. Now assume that $D$ in (B.1) is a linear homotopy functor. Then $H_{n}(Y, B ; D)=H_{n}(Y, B)$ is a homology theory on pair $_{1}$ in the sense of $(2.2)$ and we get for $s \geq 0$

$$
\begin{aligned}
E_{s, q-s}^{1} & =E_{1}^{-s, q-s}=H_{q+1}\left(U^{s+1}, U^{s}\right) \\
& =H_{q+1}\left(X \cup C X^{s}, X \cup C X^{s-1}\right) \\
& =H_{q+1}\left(\Sigma X^{s}, \Sigma X^{s-1}\right)=H_{q}\left(X^{s}, X^{s-1}\right) .
\end{aligned}
$$

Moreover by the exact sequence

$$
H_{q}\left(X^{s}\right) \rightarrow H_{q}(X) \rightarrow H_{q}\left(X / X^{s}\right) .
$$

We see that $K_{-s, q}$ in (B.1) (9) coincides with $I_{s, q-s}$ where $I_{s, q-s}$ is the image of $H_{q}\left(X^{s}\right) \rightarrow H_{q}(X)$ as on page 613 [29]. This shows that the spectral sequence (B.1) yields as a special case the Atiyah-Hirzebruch spectral sequence in XIII.3.3 [29].

\section{ApPendix C: A SPECTRAL SEQUenCE FOR SQUARE HOMOLOGY}

In this section we apply the spectral sequence of Appendix B to square homology. Let $X$ be a CW-complex with trivial 0 -skeleton $X^{0}=*$ and let $M$ be a square group. We consider the filtration, $s \geq 0$,

$$
0=K_{0, q} \subset K_{1, q} \subset \ldots \subset K_{s, q} \subset \ldots \subset H_{q}(X ; M)
$$

of square homology given by

$$
K_{s, q}=\operatorname{kernel}\left(H_{q}(X ; M) \rightarrow H_{q}\left(X / X^{s}\right)\right)
$$


where $X \rightarrow X / X^{s}$ is the quotient map. Then the spectral sequence $\left(E_{r}^{-s, q-s}, d_{r}\right)$ defined in the Appendix B converges and satisfies

$$
E_{\infty}^{-s, q-s}=K_{s, q} / K_{s-1, q} .
$$

We now determine the $E_{1}$-term of this spectral sequence. Let

$$
U^{s}=X \cup C X^{s-1} \simeq X / X^{s-1}
$$

and let $A^{s}=X^{s} / X^{s-1}$. Then we have

$$
U^{s+1}=X \cup C X^{s+1}=U^{s} \cup C A^{s}
$$

where the attaching map $A^{s} \rightarrow U^{s}$ is the composite $f: A^{s} \subset X / X^{s-1} \simeq U^{s}$ given by the inclusion. Clearly $A_{s}=M\left(C_{s}, s\right)$ is the Moore space of the free abelian group $C_{s}+H_{s}\left(X^{s}, X^{s-1} ; \mathbb{Z}\right)$ which is part of the cellular chain comlex $C_{*} X=\left(C_{*}, d\right)$. We can compute the $E_{1}$-term

$$
E_{1}^{-s, q-s}=H_{q+1}\left(U^{s+1}, U^{s} ; M\right)
$$

by applying diagram (3.4) to (C.3). This yields the following commutative diagram in which the row and the column are exact.

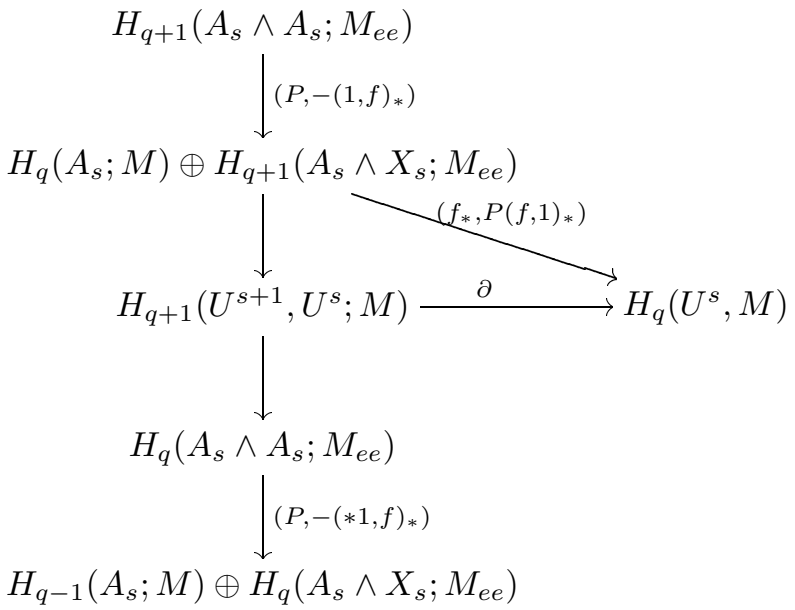

Using (7.10) this yields the following result on $H_{q+1}\left(U^{s+1}, U^{s} ; M\right)$. Let $B_{s}=$ image $\left(d: C_{s+1} \rightarrow C_{s}\right)$ be the group of boundaries in the cellular chain complex $C_{*} X$ and let $q: C_{s} \rightarrow C_{s} / B_{s}$ be the quotient map.

(C.6) Proposition. The $E_{1}$-term is given by the groups (C.4) which can be described by the following isomorphisms and exact sequences respectively.

$$
H_{q+1}\left(U^{s+1}, U^{s} ; M\right)=\left\{\begin{aligned}
0 & \text { for } \quad q<s, \\
C_{s} \otimes H_{q-s} M_{*}^{e} & \text { for } \quad s \leq q<2 s-1, \\
C_{s} \otimes H_{q-s+1}\left(X ; M_{e e}\right) & \text { for } \quad q>2 s .
\end{aligned}\right.
$$

For $q=2 s-1$ and $q=2 s$ one has the exact sequence

$$
\begin{aligned}
& 0 \rightarrow C_{s} \otimes H_{s+1}\left(X ; M_{e e}\right) \stackrel{j}{\longrightarrow} H_{2 s+1}\left(U^{s+1}, U^{s} ; M\right) \rightarrow C_{s} \otimes C_{s} \otimes M_{e e} \\
& \stackrel{\left(P,-q_{*}\right)}{\longrightarrow} \bar{C}_{s} \otimes Z_{s-1} M_{*} \oplus C_{s} \otimes C_{s} / B_{s} \otimes M_{e e} \rightarrow H_{2 s}\left(U^{s+1}, U^{s} ; M\right) \rightarrow 0 .
\end{aligned}
$$

Here $\bar{C}_{s}=C_{s}$ for $s>1$ and $\bar{C}_{1}=\pi_{1}\left(X^{1}\right)$ is the fundamental group of the 1-skeleton $X^{1}$ for $s=1$ and $Z_{0} M_{*}=M$. 
A cross effect argument shows that the inclusion $j$ in the exact sequence of (C.6) is split injective. Moreover the differential $d_{1}$ of the spectral sequence is of the form $d \otimes 1$ (with $d$ given by $C_{*} X$ ) for the groups in the first part of (C.6) with $q \neq 2 s-1,2 s$. It is interesting to compare (C.6) with the results in section 7 . This shows that the spectral sequence for $q>s$ depends only on $\hat{M}$ and hence is determined in this range by $C_{*} X$.

\section{REFERENCES}

1. Arone, G. and Mahowald, M., The Goodwillie tower of the identity functor and the unstable periodic homotopy of spheres, Preprint (1995), Northwestern University.

2. Baues, H.J., Obstruction theory, LNM 628, Springer Verlag, 1977. MR 57:7600

3. Baues, H.J., Algebraic homotopy, Cambridge University Press, 1989. MR 90i:55016

4. Baues, H.J., Metastable homotopy and homology, Quaestiones Matematicae 14, 1991, pp. 161178. MR 92j:55012

5. Baues, H.J., Quadratic functors and metastable homotopy, J. Pure Appl. Algebra 91, 1994, pp. 49-107. MR 94j:55022

6. Baues, H.J., Combinatorial Homotopy and 4-dimensional Complexes, de Gruyter, Berlin, 1991. MR 92h:55008

7. Baues, H.J., Homotopy type and homology, Oxford Mathematical Monographs, 500 pages, Oxford University Press, 1996. MR 97f:55001

8. Baues, H.J. and Conduché, D., On the 2-type of an iterated loop space, Preprint Max-PlanckInstitut für Mathematik 95-112.

9. Baues, H.J. and Hartl, M. and Pirashvili, T., Quadratic categories and square rings, J. Pure Appl. Algebra 122, 1997, pp. 1-40. CMP 98:04

10. Baues, H.J. and Pirashvili, T., Quadratic endofunctors of the catgeory of groups, Preprint MPI für Math. 95-55.

11. Baues, H.J. and Pirashvili, T., The universal coefficient theorem for quadratic functors, Preprint MPI für Math. 95-57.

12. Bousfield, A.K. and Kan, D.M., Homotopy Limits, Completions and Localizations, LNM 304 Springer Verlag, 1972. MR 51:1825

13. Cohen, F.R. and May, J.P. and Taylor, L.R., Splitting of certain spaces CX, Math. Proc. Camb. Phil. Soc., (1978), 84, pp. 465-496. MR 80a:55010

14. Conduché, D., Modules croisés généralisés de longueur 2, J. Pure and Appl. Algebra 34, 1984, pp. 155-178. MR 86g:20068

15. Curtis, E.B., Simplicial homotopy theory, Advances in Math. 6, 1971, pp. 107-209. MR 43:5529

16. Dold, A. and Puppe, D., Homologie nicht-additiver Funktoren, Anwendungen, Ann. Inst. Fourier Grenoble 11, 1961, pp. 201-312. MR 27:186

17. Goodwillie, T.G., Calculus I: the first derivative of pseudoisotopy theory, K-Theory 4, 1990, pp. 1-27. MR 92m:57027

18. Goodwillie, T.G., Calculus II: analytic functors, K-Theory 5, 1992, pp. 295-332. MR 93i:55015

19. Goodwillie, T.G., Calculus III: the Taylor series of a homotopy functor, in preparation.

20. Goodwillie, T.G., The calculus of functors, Preprint 1986 Brown University.

21. Goodwillie, T.G., Calculus I. The first derivative of a homotopy functor, Preprint Brown University.

22. Gray, B., Homotopy theory, Academic Press, 1975. MR 53:6528

23. James, J., On the suspension triad, Ann. of Math. (2) 63, 1956, pp. 191-247. MR 17:1117b

24. Johnson, B., The derivatives of homotopy theory, Trans. AMS 347, 1995, pp. 1295-1310. MR 96b:55012

25. Kan, D.M., A relation between CW-complexes and free C.s.s. groups, Amer. J. Math. 81, 1959, pp. 521-528. MR 22:1901

26. Kan, D.M., A combinatorial definition of homotopy groups, Annals of Math. 67, 1958, pp. 282312. MR 22:1897

27. Mac Lane, S., Homology, Academic Press, 1963. MR 28:122 
28. Whitehead, G.W., Recent advances in homotopy theory, AMS Regional Conference Series in Math. 5, 1970. MR 46:8208

29. Whitehead, G.W., Elements of homotopy theory, Graduate Texts in Math. 61, Springer Verlag, 1978. MR 80b:55001

Max-Planck-Institut für Mathematik, Gottfried-Claren-Strasse 26, D-53225 Bonn, GERMANY

E-mail address: bauses@mpim-bonn.mpg.de 